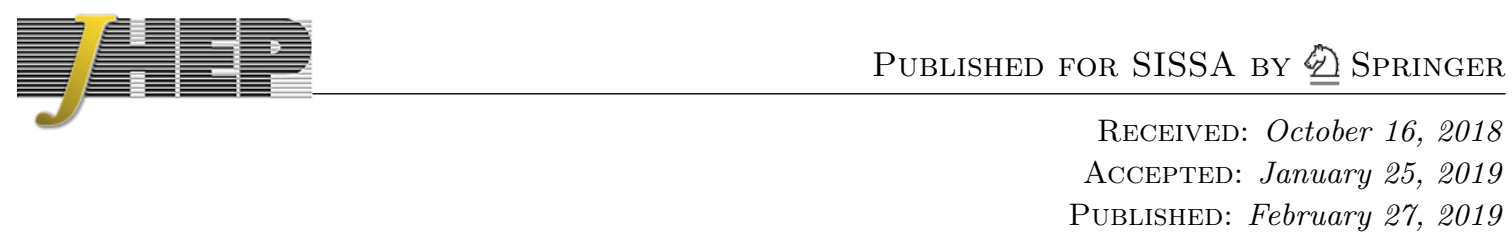

\title{
Minimally unbalanced quivers
}

\section{Santiago Cabrera, Amihay Hanany and Anton Zajac}

Theoretical Physics, The Blackett Laboratory, Imperial College London, Prince Consort Road, London, SW7 2AZ United Kingdom

E-mail: santiago.cabrera13@imperial.ac.uk, a.hanany@imperial.ac.uk, anton.zajac@imperial.ac.uk

ABSTRACT: We develop a classification of minimally unbalanced $3 d \mathcal{N}=4$ quiver gauge theories. These gauge theories are important because the isometry group $G$ of their Coulomb branch contains a single factor, which is either a classical or an exceptional Lie group. Concurrently, this provides a classification of hyperkähler cones with isometry group $G$ which are obtainable by Coulomb branch constructions. HyperKähler cones such as Coulomb branches of $3 d \mathcal{N}=4$ quivers are indispensable tools for describing Higgs branches of different theories in various dimensions. In particular, they are used to describe Higgs branches of $5 d \mathcal{N}=1 \mathrm{SQCD}$ with gauge group $\mathrm{SU}\left(N_{c}\right)$ and $6 d \mathcal{N}=(1,0) \mathrm{SQCD}$ with gauge group $\operatorname{Sp}\left(N_{c}\right)$ at the respective UV fixed points.

Keywords: Global Symmetries, Field Theories in Lower Dimensions, Supersymmetric Gauge Theory, Conformal Field Theory

ArXiv EPrint: 1810.01495v2 


\section{Contents}

1 Introduction 1

2 A $3 d$ Coulomb branch with isometry $\mathrm{SU}(n)$ and minimal set of generators

$2.1 \quad G=\mathrm{SU}(3)$

$2.2 G=\mathrm{SU}(10)$

3 A classification: minimally unbalanced quivers

4 Simply laced minimally unbalanced quivers

$4.1 G$ of type $A_{n}$

$4.2 G$ of type $D_{n}$

$4.3 G$ of type $E_{n}$

4.3.1 $G$ of type $E_{6}$

4.3.2 $G$ of type $E_{7}$

4.3.3 $G$ of type $E_{8}$

5 Non-simply laced minimally unbalanced quivers $\quad 16$

$\begin{array}{ll}5.1 G \text { of type } B_{n} & 16\end{array}$

$\begin{array}{ll}5.2 G \text { of type } C_{n} & 18\end{array}$

$\begin{array}{ll}5.3 G \text { of type } F_{4} & 20\end{array}$

$5.4 G$ of type $G_{2} \quad 21$

6 Simply laced minimally unbalanced quivers with unbalanced node connected by a non-simply laced edge

6.1 Exotic minimally unbalanced quivers with $G$ of type $A_{n}$

6.2 Exotic minimally unbalanced quivers with $G$ of type $D_{n} \quad 22$

6.3 Exotic minimally unbalanced quivers with $G$ of type $E_{n} \quad 24$

6.3.1 Exotic minimally unbalanced quivers with $G$ of type $E_{6} \quad 24$

6.3.2 Exotic minimally unbalanced quivers with $G$ of type $E_{7} \quad 24$

6.3.3 Exotic minimally unbalanced quivers with $G$ of type $E_{8} \quad 24$

7 Non-simply laced minimally unbalanced quivers with unbalanced node connected by a non-simply laced edge

7.1 Exotic minimally unbalanced quivers with $G$ of type $B_{n} \quad 27$

7.2 Exotic minimally unbalanced quivers with $G$ of type $C_{n} \quad 27$

7.3 Exotic minimally unbalanced quivers with $G$ of type $F_{4} \quad 38$

7.4 Exotic minimally unbalanced quivers with $G$ of type $G_{2}$

8 Conclusions and prospects $\quad 40$ 


\section{Introduction}

The study of the vacuum structure of SQED and SQCD with eight supercharges [1-5] constitutes a prodigious bridge between physics and mathematics. It continues the spirit of Dirac's vision on the modern role of theoretical physics [6]; the study of the geometrical properties of the vacuum moduli space rewards the researcher with the discovery of new physical phenomena. In this note we apply this principle to study the relationship between the geometry of hyperkähler ${ }^{1}$ cones [8-16] and the physics of gauge theories with eight superchagers. $^{2}$ Let us start by remembering some aspects of Supersymmetric Quantum Electrodynamics.

The Higgs branch $\mathcal{H}_{f}$ of SQED with eight supercharges, $n$ electrons, and finite gauge coupling $g$, can be computed classically [3]. It does not depend on the number of spacetime dimensions. It is a hyperkähler cone of complex dimension $2 n-2$ and it possesses an isometry under the flavor group $G=\mathrm{SU}(n)$. It is isomorphic to the reduced moduli space of one $A_{n-1}$ instanton on $\mathbb{C}^{2}$ [4]. Recent developments by Namikawa [16] show that it is actually one of the simplest hyperkähler cones with an $\mathrm{SU}(n)$ isometry, in the sense that the set of generators of the cone is minimal. ${ }^{3}$ In particular, this Higgs branch can be described as the set of all $n \times n$ complex matrices such that:

$$
\mathcal{H}_{f}=\left\{M \in \mathbb{C}^{n \times n} \mid \operatorname{tr}(M)=0, M^{2}=0 \text { and } \operatorname{rank}(M) \leq 1\right\} .
$$

This set of matrices transforms under the adjoint representation of the $\mathfrak{s l}(n, \mathbb{C})$ algebra. Given a matrix $X_{\left(2,1^{n-2}\right)}$ such that:

$$
X_{\left(2,1^{n-2}\right)}:=\left(\begin{array}{ccccc}
0 & 1 & 0 & \ldots & 0 \\
0 & 0 & 0 & \ldots & 0 \\
0 & 0 & 0 & \ldots & 0 \\
\vdots & \vdots & \vdots & \ddots & \vdots \\
0 & 0 & 0 & \ldots & 0
\end{array}\right)_{n \times n},
$$

\footnotetext{
${ }^{1}$ In this note we follow the terminology in [7] and by the notion of hyperkähler we mean symplectic and holomorphic without any statements on a metric.

${ }^{2}$ The recent work in [17] reviews the role of sigma models and supersymmetric theories on the quest for new geometrical spaces. In particular, the emphasis is given to construction of geometrical spaces with hyperkähler structure. The present note should be understood as a complimentary effort: the action of the hyperkähler quotient on an initial Lagrangian with hyperkähler geometry is replaced by the utilization of dressed monopole operators in the Coulomb branch of $3 d \mathcal{N}=4$ supersymmetric quiver gauge theory.

${ }^{3}$ By generators we mean the set of linearly independent holomorphic functions that generate the holomorphic ring of the hyperkähler cone. Remember that in a SCFT with eight supercharges the generators of the holomorphic ring of a hyperkähler branch of the moduli space (i.e. Higgs branch in any dimension or Coulomb branch in $3 d$ ) are found to be in one-to-one correspondence with chiral operators which generate the corresponding chiral ring.
} 
where $X_{\left(2,1^{n-2}\right)}$ is a block diagonal matrix, and $\left(2,1^{n-2}\right)$ indicates the presence of one elementary Jordan normal block of size 2 , and $n-2$ elementary Jordan normal blocks of size 1 , it can be acted upon by elements of the $\operatorname{group} \operatorname{PSL}(n, \mathbb{C})$. An orbit $\mathcal{O}_{\left(2,1^{n-2}\right)}$ is defined as:

$$
\mathcal{O}_{\left(2,1^{n-2}\right)}:=\left\{S \cdot X \cdot S^{-1} \mid S \in \operatorname{PSL}(n, \mathbb{C})\right\}
$$

such that $\mathcal{H}_{f}$ is isomorphic to the orbit's closure:

$$
\mathcal{H}_{f}=\overline{\mathcal{O}}_{\left(2,1^{n-2}\right)} .
$$

Note that $\left(2,1^{n-2}\right)$ is a partition of the integer number $n$. For each partition $\lambda$ of $n$, there is a matrix $X_{\lambda}$ of Jordan normal form, and its orbit $\mathcal{O}_{\lambda}$ can be defined such that its closure, $\overline{\mathcal{O}}_{\lambda}$, is a hyperkähler cone [12]. The set of all such orbits is called the set of all nilpotent orbits of $\mathfrak{s l}(n, \mathbb{C})$ and their closures are the simplest hyperkähler cones that can be built that enjoy an isometry under the group $G=\mathrm{SU}(n)$. Any other hyperkähler cone with $\mathrm{SU}(n)$ isometry that is not the closure of a nilpotent orbit of $\mathfrak{s l}(n, \mathbb{C})$ has a non-minimal ${ }^{4}$ set of generators [16].

Hyperkähler cones whose isometry $G$ contains a single factor (f.i. $\mathrm{SU}(n))$ can be classified according to grading of the generators with respect to their charge under $\mathrm{SU}(2)_{R}$, with the set of nilpotent orbits of $\operatorname{Lie}(G)$ in the simplest level of the classification. Accordingly, supersymmetric quantum field theories whose Higgs branch is a hyperkähler cone posses a similar stratification.

Three dimensional $\mathcal{N}=4$ supersymmetric gauge theories also have Coulomb branches that are hyperkähler cones. They are often related to hyperkähler $3 d$ Higgs branches via $3 d$ mirror symmetry [4]. Hence, when their isometry group $G$ has a single factor, they also admit a similar classification. Moreover, Higgs branches of $5 d \mathcal{N}=1$ and $6 d \mathcal{N}=(1,0)$ SQCD have been found to have description in terms of $3 d$ Coulomb branches when the gauge coupling $g$ is taken to infinity [18-21]. All of these recent developments suggest that a thorough classification of $3 d \mathcal{N}=4$ Coulomb branches is essential in order to carry out a systematic study of hyperkähler Higgs branches in any dimension.

Theories whose Higgs or Coulomb branches are closures of nilpotent orbits have seen an extensive analysis (see for example [22-29]). In this paper we present a classification of $3 d \mathcal{N}=4$ gauge theories that have a common property: their Coulomb branch has an isometry group $G$ which has a single factor, but need not necessarily be a closure of a nilpotent orbit of $\operatorname{Lie}(G)$. In order to adroitly produce such classification, we rely on recent advances in the study of $3 d \mathcal{N}=4$ Coulomb branches, herein denoted by $\mathcal{C}$. For $3 d \mathcal{N}=4$ theories that have an associated quiver, the isometry group $G$ of $\mathcal{C}$ has a powerful connection with the structure of the quiver. We exploit this fact, and the current understanding of dressed monopole operators $[22,30-35]$ on $\mathcal{C}$.

In section 2 we illustrate the main ideas of the paper with several examples. Section 3 presents the general method of classifying $3 d \mathcal{N}=4$ quivers such that their Coulomb branch has isometry $G$, where $G$ is any Lie group, and its set of generators is minimally extended. Sections 4 contains all cases where $G$ corresponds to a simply laced Dynkin diagram.

\footnotetext{
${ }^{4}$ This notion will be explained in more detail in the next section.
} 
Section 5 collects all cases with $G$ that corresponds to a non-simply laced Dynkin diagram. In section 6 we present an exotic extension of the classification. In particular, we consider minimally unbalanced quivers with $G$ corresponding to a simply laced Dynkin diagram but with the unbalanced node connected to the rest of the quiver via a non-simply laced edge. Section 7 contains an exotic extension of the classification of minimally unbalanced quivers with $G$ that corresponds to a non-simply laced Dynkin diagram and with the unbalanced node connected by a non-simply laced edge. Finally, section 8 offers a brief summary of the work and possible future directions in the study of minimally unbalanced supersymmetric quiver gauge theories.

\section{A $3 d$ Coulomb branch with isometry $\mathrm{SU}(n)$ and minimal set of gener- ators}

Let us start by remembering a very well known effect: the mirror symmetry of $3 d \mathcal{N}=4$ quiver gauge theories [4]. Continuing with the example from the introduction:

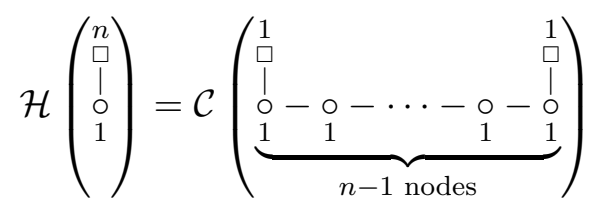

where $\mathcal{H}()$ denotes the Higgs branch of a the $3 d$ quiver at finite coupling and $\mathcal{C}()$ denotes its Coulomb branch. Note, that the gauge groups are depicted by round nodes and the flavor groups by square nodes in the quiver. Also, all the gauge groups are taken to be $\mathrm{U}(k)$, where $k$ is the label of the node. Therefore, the gauge group in the left hand side of the equation is $\mathrm{U}(1)$, and in the right hand side it is $\mathrm{U}(1)^{n-1}$. In this case, both sides of equation (2.1) are equal to the hyperkähler cone in equation (1.4), with highest weight generating function [36]:

$$
\operatorname{HWG}\left(\mu_{1}, \ldots, \mu_{n-1}, t\right)=\frac{1}{1-\mu_{1} \mu_{n-1} t^{2}},
$$

where the highest weight fugacities $\mu_{1} \mu_{n-1}$ signify that the generators of the holomorphic ring transform under the representation with highest weight $[1,0,0, \ldots, 0,0,1]$, i.e. the adjoint representation of the isometry group $\mathrm{SU}(n)$. Let us focus on the r.h.s. of equation (2.1). Note that the gauge nodes form the Dynkin diagram of Lie(SU(n)). Note also that all the gauge nodes are balanced. ${ }^{5}$ As discussed in [22], a balanced node contributes to the holomorphic ring of the Coulomb branch with polynomials of degree ${ }^{6} d=2$ (i.e. at the IR SCFT there are chiral operators $\mathcal{O}_{i}$ with conformal dimension $\left.\Delta\left(\mathcal{O}_{i}\right)=1\right)$. The set of all linearly independent holomorphic polynomials with degree $d=2$ transforms in the adjoint representation of the isometry group $G=\mathrm{SU}(n)$ [16]. This means that the number

\footnotetext{
${ }^{5}$ They satisfy $N_{f}-2 N_{c}=0$, where $N_{c}$ is the rank of the gauge node (or number of colors) and $N_{f}$ is the sum over the ranks of adjacent nodes (or number of flavors). In this particular example all gauge nodes have $N_{c}=1$ and $N_{f}=1+1=2$.

${ }^{6}$ The degree of the polynomials is represented in the highest weight generating function by the power of the fugacity $t$.
} 
of such polynomials in the example at hand is $n^{2}-1$. In [16] it is also shown that if there are only generators with degree $d=2$, then the space is the closure of a nilpotent orbit of $\mathfrak{s l}(n, \mathbb{C})=\operatorname{Lie}(G)$. Hence:

Given an isometry $G$, the set of hyperkähler cones with solely generators of degree $d=2$, which necessarily transform in the adjoint representation of $G$, is equivalent to the set of closures of nilpotent orbits of $\operatorname{Lie}(G)$.

As mentioned in the introduction, there is a one-to-one correspondence between the set of nilpotent orbits of $\mathfrak{s l}(n, \mathbb{C})$ and the set of partitions of $n$, denoted by $\mathcal{P}(n)$. Therefore, there is a finite set of hyperkähler cones with isometry $\mathrm{SU}(n)$ and minimal number of generators (i.e. $n^{2}-1$ ) transforming in the adjoint representation. Each hyperkähler cone corresponds to a different partition of $n$. Let us illustrate this using an example.

\section{$2.1 \quad G=\mathrm{SU}(3)$}

Minimal set of generator. Let the isometry group be $G=\mathrm{SU}(3)$. The set of partitions of 3 is $\mathcal{P}(3)=\{(3),(2,1),(1,1,1)\} .{ }^{7}$ There are three different hyperkähler cones corresponding to $\overline{\mathcal{O}}_{(3)}, \overline{\mathcal{O}}_{(2,1)}$ and $\overline{\mathcal{O}}_{\left(1^{3}\right)}$, respectively. For each different nilpotent orbit closure there is a corresponding $3 d \mathcal{N}=4$ quiver [22]:

$$
\begin{aligned}
& (3) \rightarrow \begin{array}{l}
3 \\
\square \\
\stackrel{0}{\circ}-\circ \\
2
\end{array}-\circ \\
& (2,1) \rightarrow \begin{array}{rrr}
1 & 1 \\
0 & 1 & 0 \\
0 & 0 \\
1 & - \\
1 & 0 \\
1
\end{array} \\
& \left(1^{3}\right) \rightarrow \stackrel{\circ}{\circ}-\circ
\end{aligned}
$$

such that

$$
\begin{gathered}
\mathcal{C}\left(\begin{array}{ll}
3 & \\
\square \\
l & \\
0 & -0 \\
2 & 1
\end{array}\right)=\overline{\mathcal{O}}_{(3)} \\
\mathcal{C}\left(\begin{array}{lr}
1 & 1 \\
\square & 0 \\
l & 1 \\
0 & 0 \\
1 & 1 \\
&
\end{array}\right)=\overline{\mathcal{O}}_{(2,1)} \\
\mathcal{C}\left(\begin{array}{ll}
\circ & 0 \\
0 & 0
\end{array}\right)=\overline{\mathcal{O}}_{\left(1^{3}\right)} .
\end{gathered}
$$

The quiver with zero rank nodes has a trivial Coulomb branch. The remaining two quivers have Coulomb branches with highest weight generating functions:

$$
\operatorname{HWG}_{(3)}\left(\mu_{1}, \mu_{2}, t\right)=\frac{1-\mu_{1}^{3} \mu_{2}^{3} t^{12}}{\left(1-\mu_{1} \mu_{2} t^{2}\right)\left(1-\mu_{1}^{3} t^{6}\right)\left(1-\mu_{2}^{3} t^{6}\right)}
$$

\footnotetext{
${ }^{7}$ In the following, we use exponential notation for partitions. For instance, partition $\{5,4,4,2,1,1,1\}$ is denoted by $\left\{5,4^{2}, 2,1^{3}\right\}$.
} 
and

$$
\operatorname{HWG}_{(2,1)}\left(\mu_{1}, \mu_{2}, t\right)=\frac{1}{1-\mu_{1} \mu_{2} t^{2}},
$$

respectively. Both Coulomb branches are solely generated by holomorphic polynomials of degree $d=2$ in the adjoint representation of $G=\mathrm{SU}(3)$, denoted by the term $\mu_{1} \mu_{2} t^{2}$ in the HWG. The degree of these polynomials, i.e. power of $t^{d}$, determines their spin $s=d / 2$ under the $\mathrm{SU}(2)_{R}$ (i.e. the R-symmetry). In this case the generators have spin $s=1$ (equivalently, the chiral ring associated with the Coulomb branch is generated by eight operators $\mathcal{O}_{i}$ in the adjoint representation of $G=\mathrm{SU}(3)$ with conformal dimension $\left.\Delta\left(\mathcal{O}_{i}\right)=1\right)$. Any other $3 d \mathcal{N}=4$ Coulomb branch $\mathcal{C}$ with isometry $\mathrm{SU}(3)$ is either isomorphic to $\mathcal{C}\left(\square_{3}-\underset{2}{\circ}-\circ\right)$ or $\mathcal{C}\left(\square-\square_{1}-\bigcirc_{1}-\square\right)$, or has extra generators $\mathcal{O}_{i}^{\prime}$ with spin $s>1$ under $\mathrm{SU}(2)_{R}$. In the latter case, the extra operators $\mathcal{O}_{i}^{\prime}$ have conformal dimension $\Delta\left(\mathcal{O}_{i}^{\prime}\right)>1$.

\section{$2.2 G=\mathrm{SU}(10)$}

Now consider the partition $\lambda=\left(2^{5}\right) \in \mathcal{P}(10)$. The quiver with Coulomb branch $\overline{\mathcal{O}}_{\left(2^{5}\right)} \subset$ $\mathfrak{s l}(10, \mathbb{C})$ takes the form:

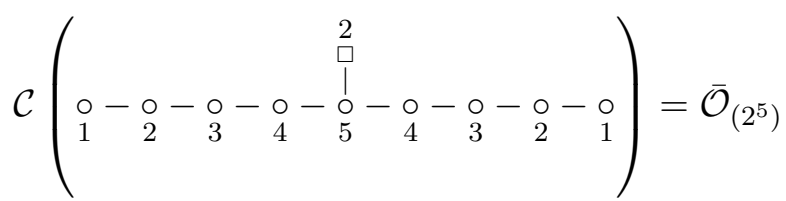

where the round and square nodes denote gauge and flavor groups, respectively. ${ }^{8}$ This Coulomb branch is minimally generated by operators $\mathcal{O}_{i}$ satisfying $\Delta\left(\mathcal{O}_{i}\right)=1$, and transforming under the adjoint representation of $\mathrm{SU}(10)$. The HWG reads:

$$
\operatorname{HWG}\left(\mu_{1}, \ldots, \mu_{9}, t\right)=\prod_{i=1}^{5} \frac{1}{1-\mu_{i} \mu_{10-i} t^{2 i}} .
$$

Extension of the minimal set of generator. Let us consider the quiver in (2.9)

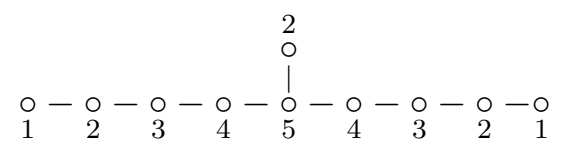

where the top gauge node is not balanced (i.e. has non-zero excess). ${ }^{9}$ Written using the Plethystic exponential (PE) [37] the HWG reads [38]:

$$
\operatorname{HWG}\left(\mu_{1}, \ldots, \mu_{9}, t\right)=\operatorname{PE}\left[\mu_{1} \mu_{9} t^{2}+\mu_{5} t^{3}+\left(1+\mu_{2} \mu_{8}\right) t^{4}+\mu_{5} t^{5}+\mu_{3} \mu_{7} t^{6}+\mu_{4} \mu_{6} t^{8}\right] .
$$

The effect of the unbalanced node on the Coulomb branch is the appearance of new operators $\mathcal{O}_{i}^{\prime}$ which are also the generators of the chiral ring. Since the conformal dimension of the new operators is $\Delta\left(\mathcal{O}_{i}^{\prime}\right)=3 / 2$, they do not modify the global symmetry of the

\footnotetext{
${ }^{8}$ In the classification of this paper, all quivers contain unitary gauge nodes and no flavor nodes.

${ }^{9}$ The excess of a node is defined as $e:=N_{f}-2 N_{c}$. If the node is balanced its excess is zero $e=0$. The excess of the top node of the quiver in equation (2.9) is: $N_{f}-2 N_{c}=5-2 \times 2=1 \neq 0$.
} 
Coulomb branch (which is only determined by the operators $\mathcal{O}_{i}$ with $\Delta\left(\mathcal{O}_{i}\right)=1$ ). Therefore the Coulomb branch of (2.9) has an isometry group which contains a single factor $G=\mathrm{SU}(10)$, but it is no longer a closure of a nilpotent orbit. This is an example of a theory that concerns the present work. In the next section we formally define the set of theories that share this property.

\section{A classification: minimally unbalanced quivers}

This section provides the answer to the main question: given a Lie group $G$, what is the set of $3 d \mathcal{N}=4$ quivers such that their Coulomb branch $\mathcal{C}$ is generated by operators $\mathcal{O}_{i}$ with $\Delta\left(\mathcal{O}_{i}\right)=1$ in the adjoint representation of $G$ (this set of operators is always present if $G$ is an isometry of $\mathcal{C}$ ) and an extra set of generators $\mathcal{O}_{i}^{\prime}$ with $\Delta\left(\mathcal{O}_{i}^{\prime}\right)>1$, such that $G$ remains the isometry of the Coulomb branch. In order to address this question, let us employ the following claim, which results from the work on monopole operators on the $3 d \mathcal{N}=4$ Coulomb branch $[22,32,33,35,39]$ : a gauge node of a $3 d \mathcal{N}=4$ quiver determines the presence of operators $\mathcal{O}_{i}$ with $\Delta\left(\mathcal{O}_{i}\right)=1$ in the Coulomb branch in the following way:

- If the node has excess $e>0$, it contributes with a single Casimir operator $\phi_{i}$, such that $\Delta\left(\phi_{i}\right)=1$.

- A set of nodes with excess $e=0$, in the form of a Dynkin diagram of a Lie group $G$, contributes with a number of operators $\mathcal{O}_{i}$ (with $\Delta\left(\mathcal{O}_{i}\right)=1$ ) equal to the dimension of $G$. There will be one Casimir operator $\phi_{i}$ per gauge node. The remaining operators are bare monopole operators $V_{i}$ that correspond to the different roots of the algebra $\operatorname{Lie}(G)$.

- If the quiver has no flavor nodes, one Casimir operator with $\Delta\left(\phi_{i}\right)=1$ needs to be removed from the counting, corresponding to the adjoint representation of the decoupled U(1) center of mass.

Two different cases of $3 d \mathcal{N}=4$ quivers with isometry $G$ on the Coulomb branch $\mathcal{C}$ can be readily identified employing this claim:

1. Nilpotent orbit's closure: $\mathcal{C}=\overline{\mathcal{O}}_{\lambda} \subset \operatorname{Lie}(G)$. All the generators of $\mathcal{C}$ have dimension $\Delta\left(\mathcal{O}_{i}\right)=1$. The gauge nodes of the quiver form the Dynkin diagram of $\operatorname{Lie}(G)$, for any classical or exceptional Lie group $G$. All gauge nodes of the quiver are balanced. Flavor nodes are added to ensure such balance condition. Moreover, the rank of the flavor nodes always follows the pattern of the weighted Dynkin diagram [12] of the corresponding nilpotent orbit $\mathcal{O}_{\lambda}$. This can realise nilpotent orbit's closures of height $\operatorname{ht}\left(\mathcal{O}_{\lambda}\right)=2 .{ }^{10}$

2. Minimally unbalanced quiver: the gauge nodes of the quiver form a minimal extension of the Dynkin diagram of $\operatorname{Lie}(G)$. By minimal extension we mean that

\footnotetext{
${ }^{10}$ The height of a nilpotent orbit is defined as in [40, section 2]. Note that for $G$ of $A$-type, this construction can be extended to nilpotent orbits of all heights $\mathrm{ht}\left(\mathcal{O}_{\lambda}\right)$, where the flavor nodes are determined by the partition $\lambda$ of the nilpotent orbit. See [22, 26, 41] for examples.
} 
there is a single extra gauge node, connected to the other gauge nodes that form the Dynkin diagram. There are no flavor nodes. All gauge nodes in the Dynkin diagram are balanced (with $e=0$ ). The the extra node is unbalanced, i.e. it has excess $e>0 .{ }^{11}$

Examples of the first case are the theories in equations (2.4) and (2.7). Equation (2.9) is an example of the second case. Both cases have a number of generators $\mathcal{O}_{i}$, with $\Delta\left(\mathcal{O}_{i}\right)=1$, equal to the dimension of $G$. In all three examples the Coulomb branch has the same isometry $G$. The difference is that the first case has no extra generators of $\mathcal{C}$, while the second case has extra generators $\mathcal{O}_{i}^{\prime}$ with $\Delta\left(\mathcal{O}_{i}^{\prime}\right)>1$. As mentioned before, $3 d \mathcal{N}=4$ quiver gauge theories whose Coulomb branches are closures of nilpotent orbits have already been extensively studied (note the recent progress for exceptional $G$ in [42]). In this note we present a classification of all minimally unbalanced quivers, for any classical Lie group $G$. We emphasize that all quivers presented in this paper are in the basic form such that the ranks are the lowest possible. Other theories can be obtained by multiplying the basic forms of the quivers by an integer number (this will not modify the isometry of the Coulomb branch).

Minimally unbalanced quiver. We are in the position to present the general solution for finding all minimally unbalanced quivers with a Coulomb branch isometry $G$, where $G$ contains a single factor. The remaining sections of the paper contain the specific results for all the different types of Lie groups. As the first step, consider a $3 d \mathcal{N}=4$ quiver $Q$ with the shape of a particular Dynkin diagram and with an extra node attached to it in the simplest fashion. ${ }^{12}$ All nodes are $\mathrm{U}\left(N_{i}\right)$ gauge nodes, where $N_{i}$ is the number of colors of the $i$-th node. The nodes in the Dynkin diagram need to be balanced (with excess $e=0$ ). In order to impose the balancing condition one can remember the vectors $\vec{v}$ and $\vec{w}$ on Nakajima's quiver varieties [13]. In this case they are used slightly differently. Let $\vec{v}$ be the vector with the ranks of all the nodes of the part of the quiver that forms the (balanced) Dynkin diagram. Let $C$ be the corresponding Cartan matrix. Then, the vector $\vec{w}$ is defined as:

$$
\vec{w}:=C \cdot \vec{v} .
$$

Note that $\vec{w}$ measures the excess in each of the nodes in $\vec{v}$ in the presence of no other nodes in the quiver. Now, one sets to zero the all components of $\vec{w}$ except of one. The non-zero component can be set to $k$. This corresponds to attaching an extra node of rank $k$ (the node that will become minimally unbalanced) at the position of the non-zero element of $\vec{w}$ and simultaneously balancing all nodes in $\vec{v}$. After fixing the rank of the imbalanced

\footnotetext{
${ }^{11}$ In the following sections the cases with $e=-1$ are discussed separately from generic cases with $e<0$. If all nodes have $e=0$, the quiver forms an affine or twisted affine Dynkin diagram of the global symmetry $G$ and these cases are also discussed separately. We refrain from discussing the pathological case of the $A_{2}^{(2)}$ twisted affine Dynkin diagram.

${ }^{12}$ Simplest fashion means that the extra node is attached by a simply laced edge to only one of the nodes of the balanced Dynkin diagram.
} 
node (node with $e \neq 0$ ) to $k$, the ranks of the balanced nodes are uniquely determined: ${ }^{13}$

$$
\vec{v}=C^{-1} \cdot \vec{w} .
$$

Finally, the value of $k$ can be chosen to be the smallest value such that all the other ranks are integer numbers. In the following sections we perform this computation for all different choices of the position of the non-zero component of $\vec{w}$. In this way, we obtain all possible minimally unbalanced quivers with a balanced subset of nodes corresponding to a certain Dynkin diagram.

\section{Simply laced minimally unbalanced quivers}

We begin our classification of minimally unbalanced quiver gauge theories with Coulomb branch isometry $G$ that corresponds to a simply laced Dynkin diagram and the unbalanced node is also connected by a simply laced edge.

\section{1 $G$ of type $A_{n}$}

Let us show one example of the approach described in the previous section. Choose $G=$ $\mathrm{SU}(9)$, with the Dynkin diagram of the form:

$$
0-0-0-0-0-0-0-0 .
$$

Let the Dynkin diagram be the balanced part of the $3 d \mathcal{N}=4$ quiver $\mathrm{Q}$. The vector $\vec{v}=\left(v_{1}, v_{2}, \ldots, v_{8}\right)$ denotes the number of colors of each node:

$$
\stackrel{\circ}{v_{1}}-\underset{v_{2}}{\circ}-\underset{v_{3}}{\circ}-\underset{v_{4}}{\circ}-\underset{v_{5}}{\circ}-\underset{v_{6}}{\circ}-\underset{v_{7}}{\circ}-\stackrel{\circ}{\circ} .
$$

Let us attach an extra node with $k$ colors to the fourth node (which has the number of colors $\left.v_{4}\right)$. This determines $\vec{w}$ :

$$
\vec{w}=(0,0,0, k, 0,0,0,0) .
$$

The resulting quiver is:

$$
\mathrm{Q}:=\underset{v_{1}}{\circ}-\underset{v_{2}}{\circ}-\underset{v_{3}}{\circ}-\stackrel{\substack{\circ \\ !}}{\circ}-\underset{v_{4}}{\circ}-\underset{v_{5}}{\circ}-\underset{v_{6}}{\circ}-\underset{v_{7}}{\circ}-\underset{v_{8}}{\circ} .
$$

Employing the Cartan matrix $C$ of $\mathrm{SU}(9)$,

$$
C:=\left(\begin{array}{cccccccc}
2 & -1 & 0 & 0 & 0 & 0 & 0 & 0 \\
-1 & 2 & -1 & 0 & 0 & 0 & 0 & 0 \\
0 & -1 & 2 & -1 & 0 & 0 & 0 & 0 \\
0 & 0 & -1 & 2 & -1 & 0 & 0 & 0 \\
0 & 0 & 0 & -1 & 2 & -1 & 0 & 0 \\
0 & 0 & 0 & 0 & -1 & 2 & -1 & 0 \\
0 & 0 & 0 & 0 & 0 & -1 & 2 & -1 \\
0 & 0 & 0 & 0 & 0 & 0 & -1 & 2
\end{array}\right),
$$

\footnotetext{
${ }^{13}$ Note that the existence of the inverse of the Cartan matrix is guaranteed since we are dealing with finite-dimensional Lie algebras.
} 
the imposed balancing condition on $v_{i}$ :

$$
\vec{v}=C^{-1} \cdot \vec{\omega}
$$

determines the ranks of the remaining nodes of the quiver. The resulting quiver takes the form:

$$
\mathrm{Q}=\underset{\frac{5 k}{9}}{\circ}-\underset{\frac{10 k}{9}}{\circ}-\underset{\substack{k \\ 0 \\ \frac{1}{9}}}{\stackrel{0}{0}}-\underset{\frac{20 k}{9}}{\circ}-\underset{\frac{16 k}{9}}{\circ}-\underset{\frac{12 k}{9}}{\circ}-\underset{\frac{8 k}{9}}{\circ}-\underset{\frac{4 k}{9}}{\circ} .
$$

The ranks of the gauge groups are integer if $k=9 p$, with $p \in \mathbb{N}$. All the nodes in the bottom row have excess $e=0$. The excess of the top gauge node with $k=9 p$ colors is:

$$
e=20 p-18 p=2 p .
$$

The lowest value of $k$ such that all other ranks $v_{i}$ are positive integers is obtained for the choice $p=1$. Therefore the quiver of interest has the form:

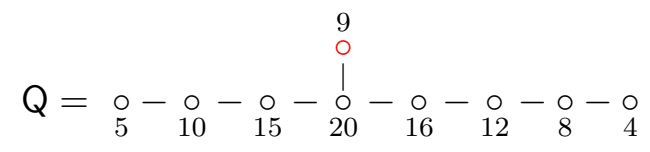

where the top node (drawn red) has excess e $=2$. Following [22], a bare monopole operator $V_{i}$ minimally charged under a node with excess $e$ has conformal dimension:

$$
\Delta\left(V_{i}\right)=(e+2) / 2 .
$$

For $e=2$ there is a bare monopole operator $V$, only charged under the magnetic dual of the unbalanced node, with dimension $\Delta(V)=2$. This is part of the set of extra generators $\mathcal{O}_{i}^{\prime}$ of the Coulomb branch. In particular, we say that all the extra generators (with $\Delta\left(\mathcal{O}_{i}^{\prime}\right)=2$ ) can be obtained by a procedure similar to that explained in [22], by turning on minimal charges of the balanced sector of the quiver. There is a total of 252 such operators, transforming in the fourth antisymmetrization of the fundamental representation of $G=\mathrm{SU}(9)$, denoted by Dynkin labels $[0,0,0,1,0,0,0,0]$, and its complex conjugate representation $[0,0,0,0,1,0,0,0] .{ }^{14}$

The various choices of $\vec{w}$ produce different quivers $Q$ where the extra node is attached either to the fourth, the third, the second or the first node in the Dynkin diagram of $G=\mathrm{SU}(9) .{ }^{15}$ In each case $k$ is chosen to be the smallest value such that the rest of the ranks are positive integers. The different results and the excess of the extra node are depicted in table 1. It is crucial to distinguish four cases based on the excess of the unbalanced node which in turn determines the presence of extra operators with various values of conformal dimension. Following the terminology of [22], we summarize the possible types of theories in table 2. This terminology is used throughout this paper.

\footnotetext{
${ }^{14}$ Note that the appearance of the 252 new operators can be read off the quiver since the unbalanced node is attached to the fourth Dynkin node, indicating that the extra generators transform in this representation and its complex conjugate representation.

${ }^{15}$ The quiver in figure 1 enjoys outer $\mathbb{Z}_{2}$ automorphism symmetry therefore other choices of $\vec{w}$ yield equivalent quivers to those already included.
} 


\begin{tabular}{|c|c|c|}
\hline Quiver & Excess & Type \\
\hline 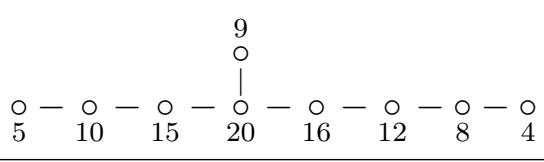 & 2 & Good \\
\hline 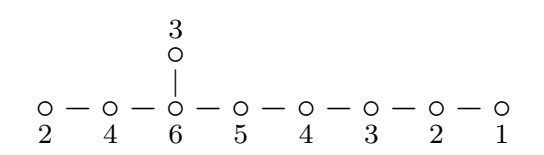 & 0 & Good \\
\hline 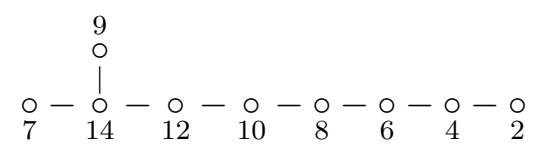 & -4 & $\mathrm{Bad}$ \\
\hline $\begin{array}{l}9 \\
\circ \\
\text { ! } \\
\circ \\
8\end{array}-\underset{7}{\circ}-\underset{6}{\circ}-\underset{5}{\circ}-\underset{4}{\circ}-\underset{3}{\circ}-\underset{2}{\circ}-\stackrel{\circ}{1}$ & -10 & Bad \\
\hline
\end{tabular}

Table 1. Quivers with a balanced $A_{8}$ subset and a single unbalanced node.

\begin{tabular}{|c|c|c|}
\hline Excess & Type of the theory & $\Delta$ of extra generators \\
\hline$e>0$ & Good & $\Delta \geq 1$ \\
\hline$e=0$ & Good & $\Delta=1$ \\
\hline$e=-1$ & Ugly & $\Delta>0$ \\
\hline$e<-1$ & Bad & not applicable \\
\hline
\end{tabular}

Table 2. Types of minimally unbalanced quiver gauge theories based on excess of the extra node.

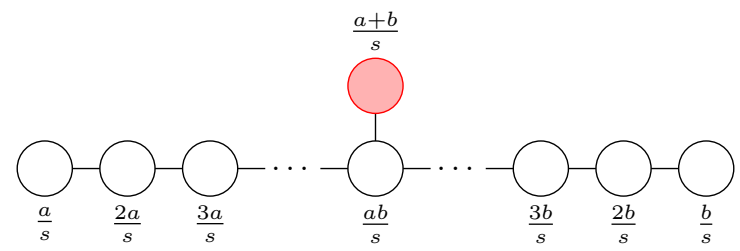

Figure 1. Generic quiver with $G=\mathrm{SU}(n)$ global symmetry, with $n=a+b$, where $a, b \in \mathbb{N}$ and $s$ is the greatest common divisor of $a$ and $b$. The excess of the bottom nodes is $e=0$. The excess of the top node is $e=(a b-2 a-2 b) / s$. We are interested in the subset of quivers with $(a b-2 a-2 b) / s \neq 0$. The quaternionic dimension of the Coulomb branch of the quiver can be expressed using parameters $a$ and $b: \operatorname{dim}_{\mathbb{H}}=\frac{(a b+2)(a+b)}{2 s}-1$.

Hence the first and the second row in table 1 contain good theories that are: unbalanced with positive excess and fully balanced, respectively. The third and fourth row in table 1 contain bad quivers that are both unbalanced with negative excess.

General cas. All quivers $Q$ that can be obtained with this procedure are summarized by a two parameter family, depicted in figure 1 . The quiver in figure 1 contains $a+b$ gauge nodes, of which $a+b-1$ are balanced. The remaining unbalanced node (conveniently drawn 
red thorough this work) has excess:

$$
e(a, b)=\frac{a b-2 a-2 b}{\operatorname{gcd}(a, b)}
$$

where $\operatorname{gcd}(a, b)$ denotes the greatest common divisor of $a$ and $b$. For $e(a, b)>0$ the global symmetry of the Coulomb branch is $\mathrm{SU}(n)$, where $n=a+b$, and one says that the quiver is minimally unbalanced with positive excess. Therefore, the quiver in the first row in table 1 with $e(5,4)=2$ corresponds to a good theory with positive excess. The quiver in the second row with $e(2,1)=0$ represents a good theory that is fully balanced since all nodes have excess zero. The two bad theories with $e(7,2)=-4$ and $e(0,1)=-10$ are contained in the third and the fourth row of table 1, respectively. Minimally unbalanced quivers with the unbalanced node with excess $e=-1$ have either the entire or a part of the Coulomb branch freely generated. ${ }^{16}$ Equation (4.10) defines a function:

$$
\begin{aligned}
e: \mathbb{N} \times \mathbb{N} & \rightarrow \mathbb{Z} \\
(a, b) & \mapsto e(a, b)
\end{aligned}
$$

that maps the two parameters of the family $a$ and $b$ to the excess of the top node of the corresponding quiver. This function can be visualized by defining a matrix $M$, with elements:

$$
M_{a b}=e(a, b) .
$$

Let $a$ and $b$ run from 1 to 16 , then $M$ is $16 \times 16$ :

$$
M=\left(\begin{array}{ccccccccccccccccc}
-3 & -4 & -5 & -6 & -7 & -8 & -9 & \mathbf{- 1 0} & -11 & -12 & -13 & -14 & -15 & -16 & -17 & -18 \\
-4 & -2 & -4 & -2 & -4 & -2 & \mathbf{- 4} & -2 & -4 & -2 & -4 & -2 & -4 & -2 & -4 & -2 \\
-5 & -4 & -1 & -2 & -1 & \mathbf{0} & 1 & 2 & 1 & 4 & 5 & 2 & 7 & 8 & 3 & 10 \\
-6 & -2 & -2 & 0 & \mathbf{2} & 2 & 6 & 2 & 10 & 6 & 14 & 4 & 18 & 10 & 22 & 6 \\
-7 & -4 & -1 & \mathbf{2} & 1 & 8 & 11 & 14 & 17 & 4 & 23 & 26 & 29 & 32 & 7 & 38 \\
-8 & -2 & \mathbf{0} & 2 & 8 & 2 & 16 & 10 & 8 & 14 & 32 & 6 & 40 & 22 & 16 & 26 \\
-9 & -4 & 1 & 6 & 11 & 16 & 3 & 26 & 31 & 36 & 41 & 46 & 51 & 8 & 61 & 66 \\
-\mathbf{1 0} & -2 & 2 & 2 & 14 & 10 & 26 & 4 & 38 & 22 & 50 & 14 & 62 & 34 & 74 & 10 \\
-11 & -4 & 1 & 10 & 17 & 8 & 31 & 38 & 5 & 52 & 59 & 22 & 73 & 80 & 29 & 94 \\
-12 & -2 & 4 & 6 & 4 & 14 & 36 & 22 & 52 & 6 & 68 & 38 & 84 & 46 & 20 & 54 \\
-13 & -4 & 5 & 14 & 23 & 32 & 41 & 50 & 59 & 68 & 7 & 86 & 95 & 104 & 113 & 122 \\
-14 & -2 & 2 & 4 & 26 & 6 & 46 & 14 & 22 & 38 & 86 & 8 & 106 & 58 & 42 & 34 \\
-15 & -4 & 7 & 18 & 29 & 40 & 51 & 62 & 73 & 84 & 95 & 106 & 9 & 128 & 139 & 150 \\
-16 & -2 & 8 & 10 & 32 & 22 & 8 & 34 & 80 & 46 & 104 & 58 & 128 & 10 & 152 & 82 \\
-17 & -4 & 3 & 22 & 7 & 16 & 61 & 74 & 29 & 20 & 113 & 42 & 139 & 152 & 11 & 178 \\
-18 & -2 & 10 & 6 & 38 & 26 & 66 & 10 & 94 & 54 & 122 & 34 & 150 & 82 & 178 & 12
\end{array}\right)
$$

The elements in bold are those that correspond to the quivers of length $a+b-1=8$, i.e. those in table 1 . One can see that for a generic quiver the excess is positive. A theory

\footnotetext{
${ }^{16}$ See observation 3.1 in [43].
} 
with $a+b-1>8$ is $b a d$ (negative excess) only if one of the two parameters is either 1 or 2. Furthermore, there are only three cases where the extra node is also balanced, i.e. excess $e(a, b)=0$. These are: $(a, b)=(3,6),(a, b)=(4,4)$ and $(a, b)=(6,3)$. The first and last cases correspond to an enhancement of the global symmetry of the Coulomb branch from $\mathrm{SU}(9)$ to $E_{8}$. The case $(a, b)=(4,4)$ sees a similar enhancement, this time from $\mathrm{SU}(8)$ to $E_{7}$. The three cases with $e=-1$ are obtained for $(a, b) \in\{(3,3),(3,5),(5,3)\}$. For $(a, b)=(3,3)$ the greatest common divisor is $\operatorname{gcd}(3,3)=3$, therefore, the quiver takes the form:

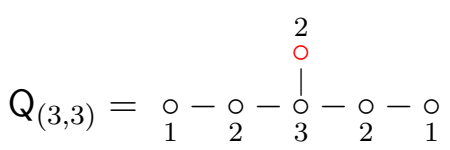

and the Coulomb branch of this quiver is a freely generated variety (see 3.10 in [21]): ${ }^{17}$

$$
\mathcal{C}=\mathbb{H}^{10} .
$$

For $(a, b)=(3,5)$ (or equivalently $(a, b)=(5,3))$ the quiver takes the form:

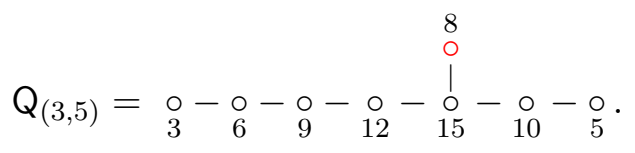

The quaternionic dimension of the Coulomb branch in 4.16 is 67 . The unbalanced node connects to the Dynkin node that corresponds to the $\mathrm{SU}(8)$ representation with Dynkin labels $[0,0,0,0,1,0,0]$ and with dimension 56. Drawing intuition from the quiver in 4.14 one would expect 112 new operators transforming in the $[0,0,0,0,1,0,0]$ and its complex conjugate rep $[0,0,1,0,0,0,0]$. Although the excess is $e=-1$ (i.e. same as in freely generated 4.14) the Coulomb branch of 4.16 seems to be more complicated (i.e. has both a freely generated as well as a non-trivial part) and we leave its explicit computation for future study.

A formula for the HWG for minimally unbalanced $A$-type quivers with $a=b, s=1$ (i.e. with outer $\mathbb{Z}_{2}$ automorphism symmetry) is given by equation (23) in [38]. Quivers of this type also show up in the study of Higgs branches of $5 d \mathcal{N}=1$ theories with 8 supercharges [18].

\section{2 $G$ of type $D_{n}$}

Let us turn our focus to minimally unbalanced quivers with Coulomb branch isometry $G=\mathrm{SO}(2 n)$. The Dynkin diagram of $\mathfrak{s o}(2 n)$ is of the form:

$$
\circ-\circ-0-\cdots-\circ-\stackrel{\circ}{i}-0 .
$$

We find a two parameter family $a, n$, where $a$ is the position of the extra node starting from the left, and $n$ is the total number of balanced nodes. Based on whether the unbalanced

\footnotetext{
${ }^{17}$ Since the balanced sub-quiver corresponds to $A_{5}$ global symmetry, but $\mathbb{H}^{10}$ has isometry $\operatorname{Sp}(10)$, we find an embedding: $\mathrm{SU}(6) \hookleftarrow \mathrm{Sp}(10)$. In particular, the pseudo-real fundamental rep of $\mathrm{Sp}(10)$ projects to the pseudo-real $3^{\text {rd }}$ rank antisymmetric rep of $\mathrm{SU}(6):[1,0,0,0,0]_{\mathrm{Sp}(10)} \hookrightarrow[0,0,1,0,0]_{\mathrm{SU}(6)}$.
} 
node attaches to one of the nodes on the main chain (i.e. $a<n-1)$ or to one of the spinor nodes (i.e. $a=n$ ) we distinguish two categories with two further sub-categories:

- Unbalanced node attached to a node on the main chain:

- Unbalanced node of rank 1 connects to an even node and the total number of balanced nodes is either even or odd. This family of quivers is contained in the first row in table 3.

- Unbalanced node of rank 2 connects to an odd node and the total number of balanced nodes is either even or odd. This family of quivers is depicted in the second row of table 3 .

- Unbalanced node attached to one of the spinor nodes:

- Unbalanced node is of rank 2 and the total number of balanced nodes is even. This family of quivers is depicted in the third row in table 3.

- Unbalanced node is of rank 4 and the total number of balanced nodes is odd. This family of quivers is contained in the fourth row in table 3.

Note that the excess depends on a single parameter $a$. It is given by linear equations shown in the third column in table 3.

Note that we find the following special cases:

- In the first row:

- for $a=2$ the quiver has zero excess and corresponds to the reduced moduli space of one $D_{n}$ instanton on $\mathbb{C}^{2}$.

- In the third row:

- for $m=4$ one obtains the affine $E_{8}$ Dynkin diagram corresponding to the reduced moduli space of one $E_{8}$ instanton on $\mathbb{C}^{2}$. The Coulomb branch is denoted as $\mathcal{C}=\overline{\min _{E_{8}}}$.

- In the last row:

- for $m=3$ (or equivalently $n=7$ ) one obtains a peculiar quiver with $e=-1$ of the form:

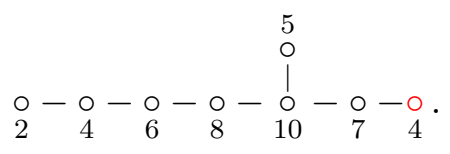

Similarly as for the quiver in 4.16 the unbalanced node does not connect to a node corresponding to a pseudo-real representation. In the case of 4.17 , the unbalanced node has negative excess $e=-1$ and it connects to a Dynkin node corresponding to the complex spinor representation with dimension 64 . The dimension of the Coulomb branch is $45 .{ }^{18}$ Analogically with 4.16 , it seems that

\footnotetext{
${ }^{18}$ Recall that the quaternionic dimension of the Coulomb branch can be read off from the quiver as: $\operatorname{dim}(\mathcal{C})=\sum_{i} r_{i}-1$, where $r_{i}$ denotes the rank of a particular node.
} 


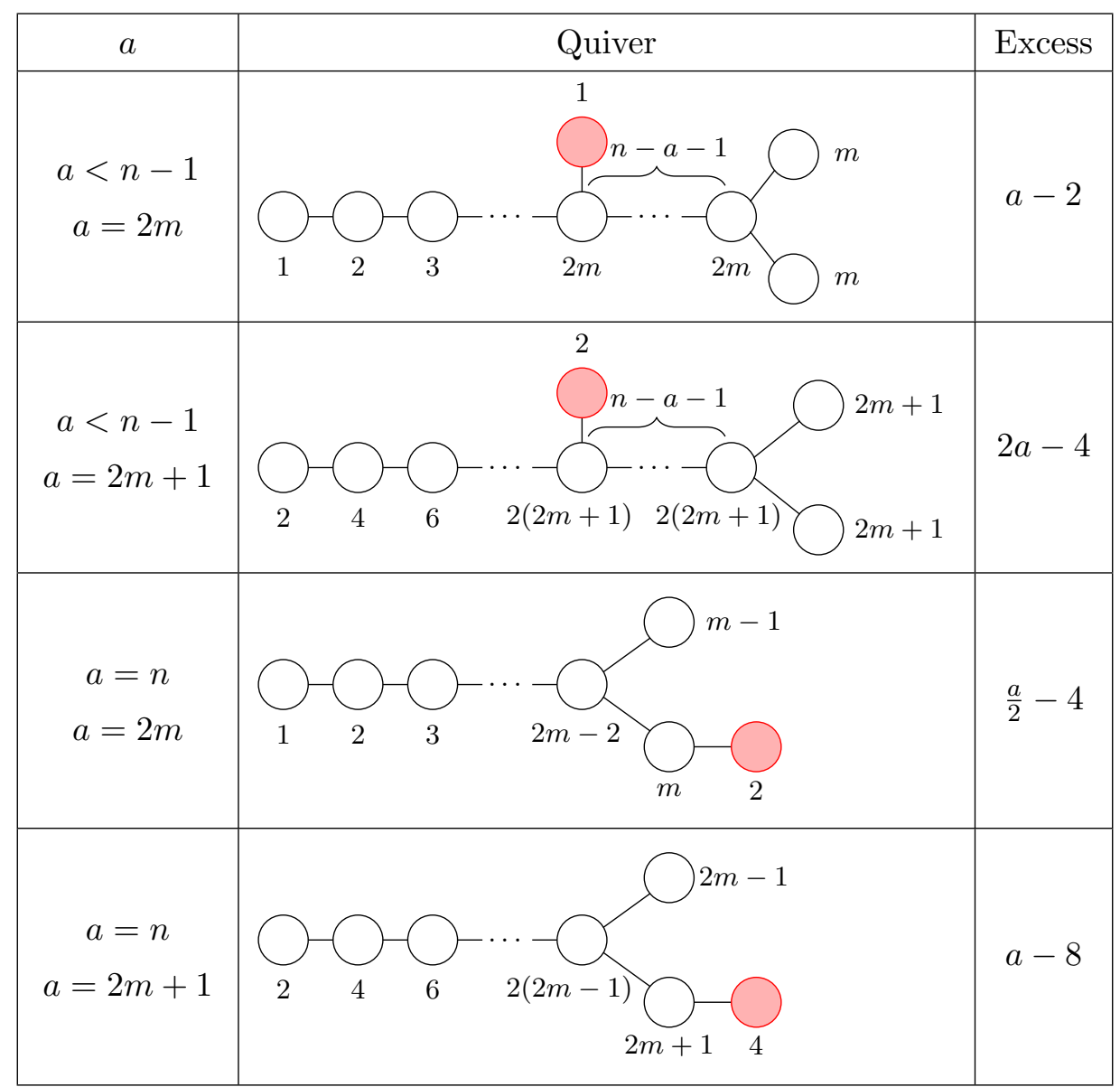

Table 3. Classification of minimally unbalanced quivers with $G=\mathrm{SO}(2 n)$.

the Coulomb branch is rather complicated with both a freely generated as well as a non-trivial part. The explicit computation of $\mathcal{C}$ is left for future study.

The HWG for the case in the third row in table 3 is given using $D_{n}$ highest weight fugacities by equation (26) in [38].

\section{3 $G$ of type $E_{n}$}

Let us finally proceed by analyzing the last category of simply laced theories with $G$ of type $E_{n}$. All the different minimally unbalanced quivers with a certain $E$-type exceptional global symmetry can be written down explicitly. We report the excess of the unbalanced node in the second column of the classification tables. Note that all the quivers in the classification that are balanced (extra node drawn orange) are the affine Dynkin diagrams of the corresponding global symmetry, where the affine node has rank 1 . When such node is taken to be a flavor node, the quiver corresponds to both the closure of the minimal nilpotent orbit of $\mathfrak{e}_{n}$ algebra, and to the reduced moduli space of one $E_{n}$ instanton on $\mathbb{C}^{2}[4,35]$. 


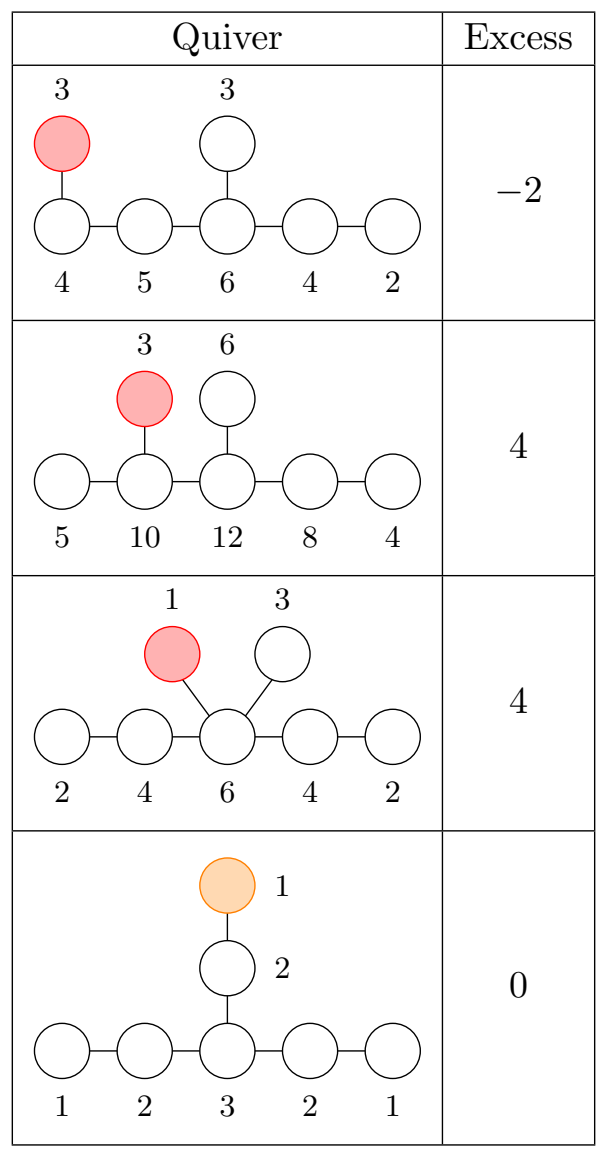

Table 4. Minimally unbalanced quivers with $G=E_{6}$.

\subsection{1 $G$ of type $E_{6}$}

For $G=E_{6}$ one explicitly writes down all the cases as displayed in table 4 . Note that there are only four distinct cases due to the $\mathbb{Z}_{2}$ outer automorphism of the $E_{6}$ Dynkin diagram. Also note that when the extra node happens to be balanced, it is drawn orange. This convention is used throughout the paper. The last row in table 4 is special (with excess $e=0$ ) and its Coulomb branch is the reduced moduli space of one $E_{6}$ instanton on $\mathbb{C}^{2}[4,35]$. The HWG for this quiver is given in terms of the $\mathrm{SU}(6) \times \mathrm{SU}(2)$ highest weight fugacities by equation (28) in [38].

\subsection{2 $G$ of type $E_{7}$}

Next, we consider minimally unbalanced quivers with $E_{7}$ global symmetry. One proceeds by attaching the unbalanced node from leftmost to the rightmost node. Due to the lack of any automorphism of the $E_{7}$ Dynkin diagram one has to exhaust all 7 cases. The resulting minimally unbalanced quivers are collected in table 5 . The first row in table 5 is again a special case since its Coulomb branch is a reduced moduli space of one $E_{7}$ instanton on $\mathbb{C}^{2}[4,44]$. The HWG for this theory is given in terms of $\mathrm{SU}(8)$ highest weight fugacities, by equation (44) in [38]. The last row of table 5 depicts a theory with excess $e=-1$. The 
Coulomb branch is freely generated:

$$
\mathcal{C}=\mathbb{H}^{28}
$$

and we find the embedding $[1,0,0,0,0,0,0]_{E_{7}} \hookleftarrow[1,0,0,0,0,0,0]_{\operatorname{Sp}(28)}$ of $E_{7}$ inside $\operatorname{Sp}(28)$. Both of these 56 dimensional representations are pseudo-real which is consistent with the expectation from $\mathbb{H}^{28}$. More generally, $\mathbb{H}^{n}$ is always generated by $2 n$ generators that transform under the pseudo-real fundamental representation of $\operatorname{Sp}(n)$.

\subsection{3 $G$ of type $E_{8}$}

Finally, we present all minimally unbalanced theories with global symmetry $G=E_{8}$ in tables 6 and 7. Again, we exhaust all eight distinct cases. The quiver in the shape of an affine $E_{8}$ Dynkin diagram in the last row of table 7 is readily identified as the moduli space of one $E_{8}$ instanton on $\mathbb{C}^{2}[4,44]$. In terms of the $\mathrm{SO}(16)$ highest weight fugacities, the HWG for this quiver is given by equation (142) in [38].

\section{Non-simply laced minimally unbalanced quivers}

In this section, we use the methods of section 3 to classify all minimally unbalanced quivers with a global symmetry that corresponds to a non-simply laced Dynkin diagram and the unbalanced node connected to the rest of the quiver via a simply laced edge. By the sequel, this section contains minimally unbalanced quivers of $B C F G$-series. The excess of the unbalanced node is shown in a separate column in all classification tables.

\section{$5.1 G$ of type $B_{n}$}

Analogically to $D$-type, minimally unbalanced quivers with $\mathrm{SO}(2 n+1)$ global symmetry divide into cases based on two parameters, $a$ and $n$, where $a$ is the position of the extra unbalanced node and $n$ is the total number of balanced nodes which are in the shape of a $B$-type Dynkin diagram. Similarly to $\mathrm{SO}(2 n)$, there are four different cases, collected in table 8. In the first case, the unbalanced node is attached to the $2 m$-th node from the left, the rank of the unbalanced node is 1 and the total number of balanced nodes is either even or odd. In the second case, the rank 2 unbalanced node attaches to the $(2 m+1)$-th node from the left and the total number of balanced nodes is either even or odd. The first two cases are contained in the first and the second row of table 8, respectively. When the unbalanced node attaches to the last spinor node of the Dynkin diagram we distinguish two cases. Either the unbalanced node has rank 1 and the total number of balanced nodes is even, or the unbalanced node is of rank 2 and the total number of balanced nodes is odd. These two cases are shown is the third and fourth row in table 8 , respectively. The excess is given in the last column of table 8 in terms of $a$.

Lets discuss the special cases:

- In the first row:

- for $a=2$ the quiver is the affine Dynkin diagram of $B_{n}$, the excess is zero, and we write $\mathcal{C}=\overline{\min _{B_{n}}}$ (i.e. the Coulomb branch is the closure of the minimal 


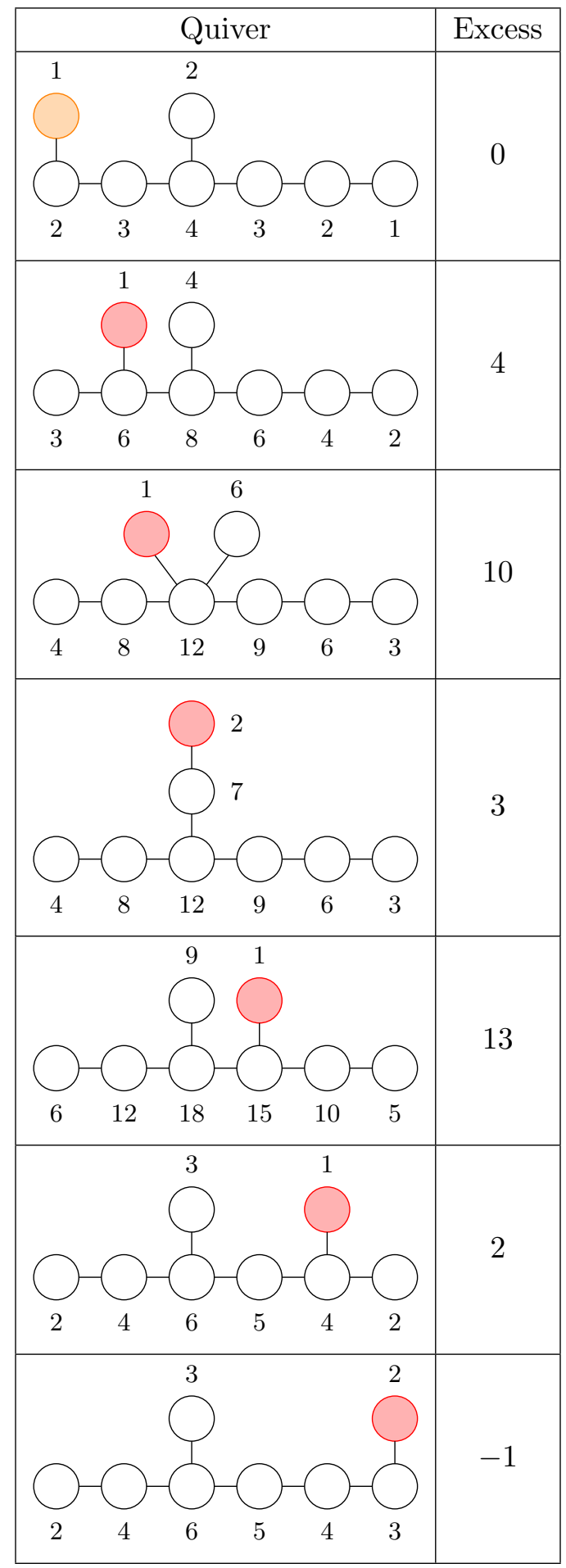

Table 5. Minimally unbalanced quivers with $G=E_{7}$. 


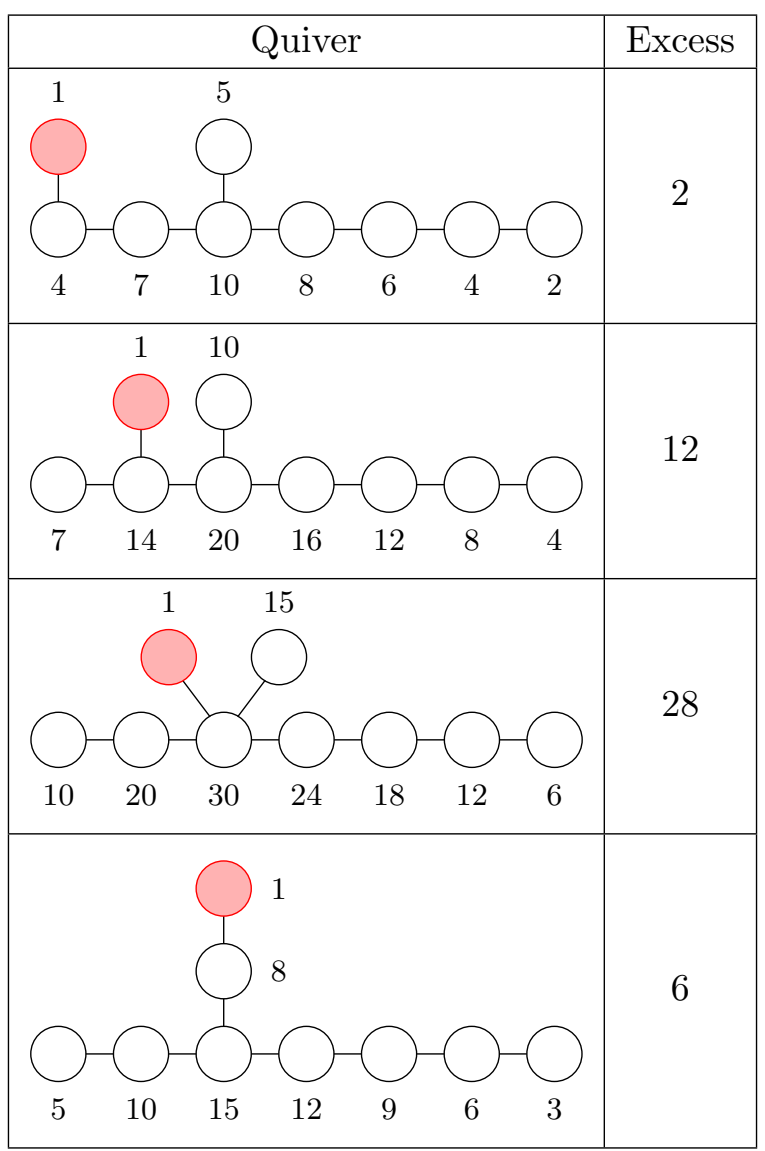

Table 6. First set of minimally unbalanced quivers with $G=E_{8}$.

nilpotent orbit of $\mathrm{SO}(2 n+1)$ or alternatively one says that is isomorphic to the reduced moduli space of one $B_{n}$ instanton on $\mathbb{C}^{2}[39]$ ).

- In the third row:

- for $m=1$ one obtains the $C_{2}$ Dynkin diagram with an unbalanced node with $e=-1$. When the unbalanced node is ungauged we have $\mathcal{C}=\mathbb{H}^{2}$ (i.e. a freely generated Coulomb branch).

- for $m=2$ one obtains the affine $F_{4}$ Dynkin diagram. Assuming the leftmost node is ungauged we have $\mathcal{C}=\overline{\min _{F_{4}}}$ (see section 5.3).

- In the fourth row:

- for $m=1$ one obtains the $B_{3}$ Dynkin diagram with an unbalanced node with excess $e=-1$ connected to the spinor node. The spinor rep of $\mathrm{SO}(7)$ is real and therefore we expect $\mathcal{C}$ to be freely generated.

\section{$5.2 G$ of type $C_{n}$}

Next, we classify all minimally unbalanced quivers with $\operatorname{Sp}(n)$ global symmetry. We employ the same two parameters $n$, number of balanced nodes in form of the C-type Dynkin 


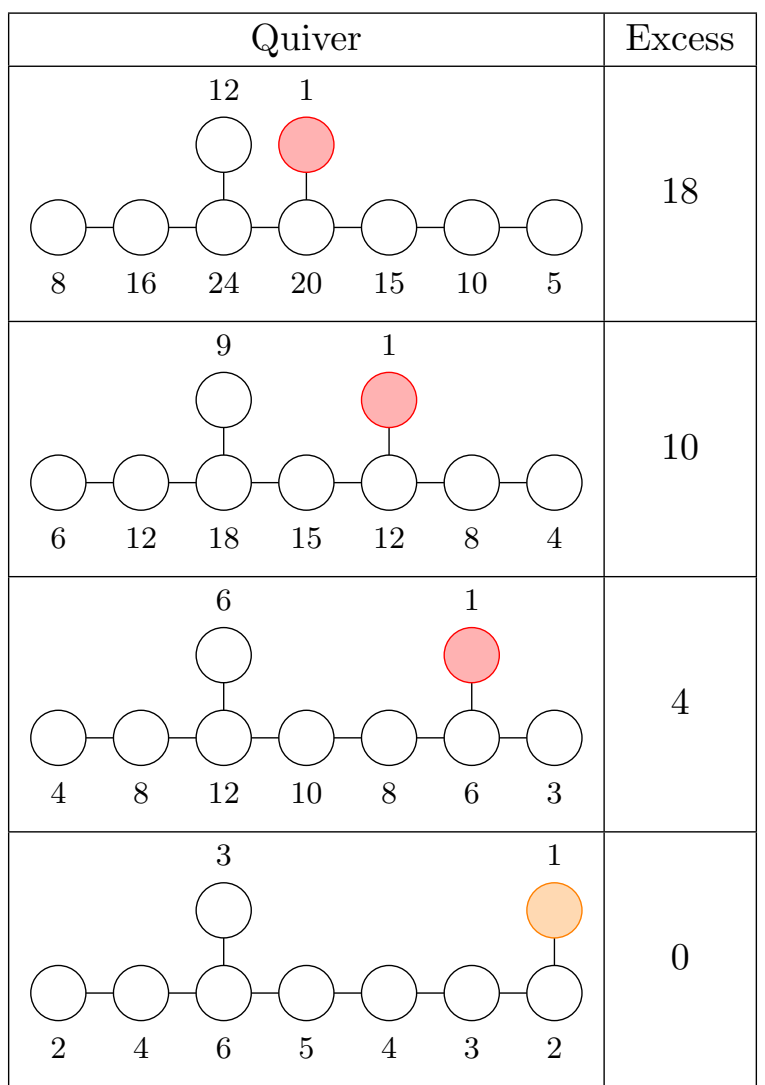

Table 7. Second set of minimally unbalanced quivers with $G=E_{8}$.

diagram, and $a$, position of the unbalanced node from the left. There are two different cases based on the position of the unbalanced node, see table 9 . In the first row of table 9 we show the case where the unbalanced node is not attached to the rightmost balanced node $(a<n)$. If the unbalanced node is connected to the rightmost node $(a=n)$, it is of rank 2 and the resulting quiver is depicted in the second row of table 9 . In both cases the total number of balanced nodes is either even or odd.

Let us now look at the special cases:

- In the first row: for $a=2$ the excess is zero and the quiver is the $A_{2 k-1}^{(2)}$ twisted affine Dynkin diagram, where $2 k-1=n+1$.

- In the second row:

- for $a=3$ one obtains the $C_{3}$ Dynkin diagram with an unbalanced node connected to the rightmost node and with excess $e=-1$. Given the value of excess and the fact that the unbalanced node connects to the pseudo-real representation denoted by $[0,0,1]_{C_{3}}$ one expects that the Coulomb branch is freely generated, namely $\mathcal{C}=\mathbb{H}^{7}$. Indeed, this claim is confirmed by a computation.

- for $a=4$ one obtains the Dynkin digram of the twisted affine $E_{6}^{(2)}$ algebra with zero excess. 


\begin{tabular}{|c|c|c|c|}
$a<n$ \\
\hline$a=2 m$ \\
$a=2 m+1$
\end{tabular}

Table 8. Classification of minimally unbalanced quivers with $G=\mathrm{SO}(2 n+1)$.

\begin{tabular}{|c|c|c|}
\hline$a<n$ \\
\hline$a=n$
\end{tabular}

Table 9. Classification of minimally unbalanced quivers with $G=\operatorname{Sp}(n)$.

\section{$5.3 G$ of type $F_{4}$}

Let us now classify all minimally unbalanced quivers with $F_{4}$ global symmetry on their Coulomb branch. Starting form the Dynkin diagram of $F_{4}$ and employing the methods of section 3 one obtains the four cases depicted in table 10. Note that the first and the last row of table 10 contain quivers that are balanced. The Coulomb branch of the quiver in the first row is the reduced moduli space of one $F_{4}$ instanton on $\mathbb{C}^{2}$ [39]. The Coulomb branch of the quiver in the last row is the closure of the next to minimal nilpotent orbit of $\mathfrak{f}_{4}$ algebra [42]. 


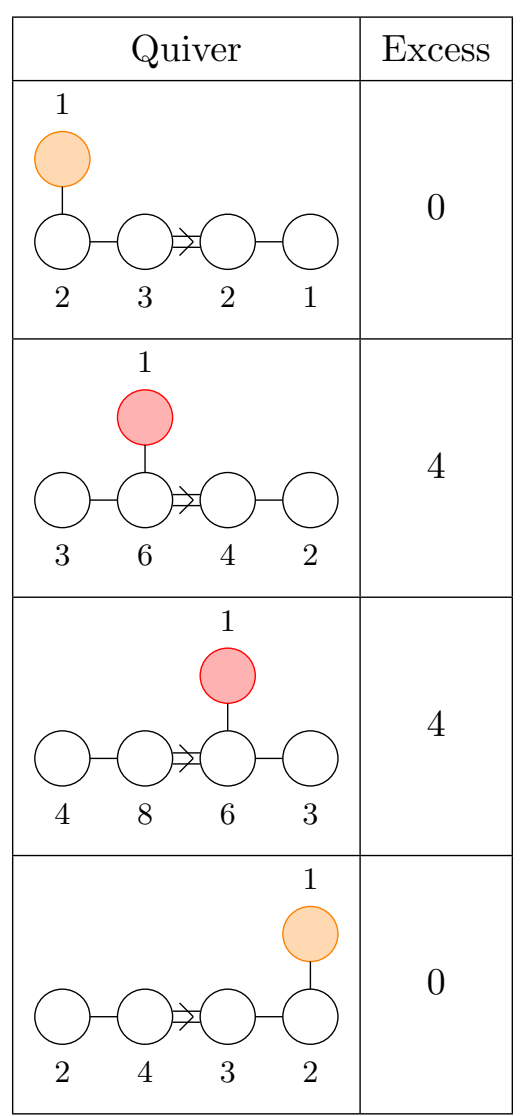

Table 10. Classification of minimally unbalanced quivers with $G=F_{4}$.

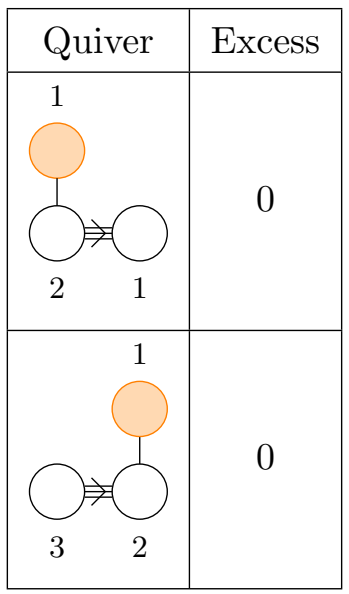

Table 11. Classification of minimally unbalanced quivers with $G=G_{2}$.

\section{$5.4 G$ of type $G_{2}$}

Finally, applying the methods of section 3 to a $G_{2}$ Dynkin diagram yields two cases of minimally unbalanced quiver theories with $G_{2}$ global symmetry on their Coulomb branch. The two quivers are depicted in table 11. Note that both cases are balanced and the first row contains the reduced moduli space of one $G_{2}$ instanton on $\mathbb{C}^{2}$ [39]. 


\section{Simply laced minimally unbalanced quivers with unbalanced node con- nected by a non-simply laced edge}

In this section, we classify all minimally unbalanced quivers with the unbalanced node connected to the rest of the quiver with a double or triple laced edge, such that the balanced subset of nodes forms a finite simply laced Dynkin diagram. We term this part of the classification exotic since it is the first time these quivers appear and the systematic study of the field theoretic aspects of these quiver gauge theories is yet to be done. The first level of distinction in the following classification is based on the Dynkin type of the balanced subset of nodes. Further levels of distinction are the following:

- Type of the non-simply laced edge:

- Double Edge

- Triple Edge

- Position of the unbalanced node

- Direction of the non-simply laced edge with respect to the unbalanced node

- Outwards from the unbalanced node

- Inwards to the unbalanced node

Let us begin the exotic classification with $A$-type minimally unbalanced quivers with the unbalanced node connected by a double or triple laced edge.

\subsection{Exotic minimally unbalanced quivers with $G$ of type $A_{n}$}

Tables 12 and 13 collect all exotic minimally unbalanced quivers of $A_{n}$ type. Table 12 contains quivers where the unbalanced node attaches via a double laced edge. Table 13 depicts quivers with the unbalanced node attached by a triple laced edge.

\subsection{Exotic minimally unbalanced quivers with $G$ of type $D_{n}$}

Let us now classify the exotic minimally unbalanced quivers with $G=\mathrm{SO}(2 n)$. First level of distinction is based on whether the unbalanced node connects via a double or triple laced edge. Second level of distinction is based on the orientation of the non-simply laced edge with respect to the unbalanced node (i.e. inwards or outwards). Finally, we distinguish cases based on whether the unbalanced node attaches to one of the nodes on the main chain or to one of the spinor nodes. ${ }^{19}$ The results of the classification are divided into two tables. Tables 14 and 15 collect the results for exotic minimally unbalanced quivers of $D$-type with the unbalanced node connected to the rest of the quiver with a double laced edge. Exotic $D$-type minimally unbalanced quivers with the unbalanced node connected by a triple laced edge are reported in tables 16 and 17 .

\footnotetext{
${ }^{19}$ Attaching the unbalanced node to the co-spinor node instead of the spinor node yields equivalent cases to those already included.
} 


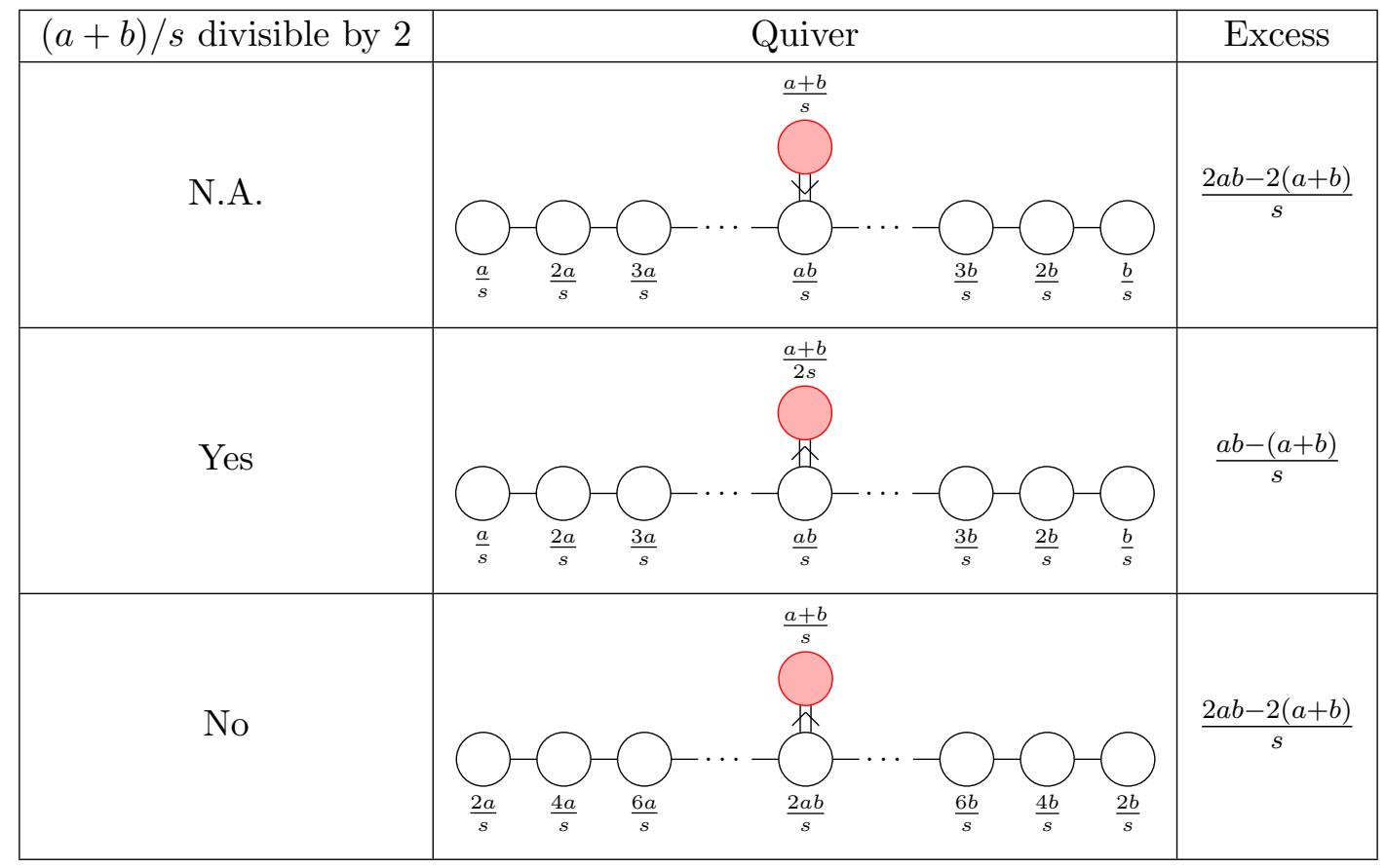

Table 12. Exotic minimally unbalanced quivers with $G=\mathrm{SU}(n), n=a+b$ and a double laced edge. $s$ is the greatest common divisor of $a$ and $b$.

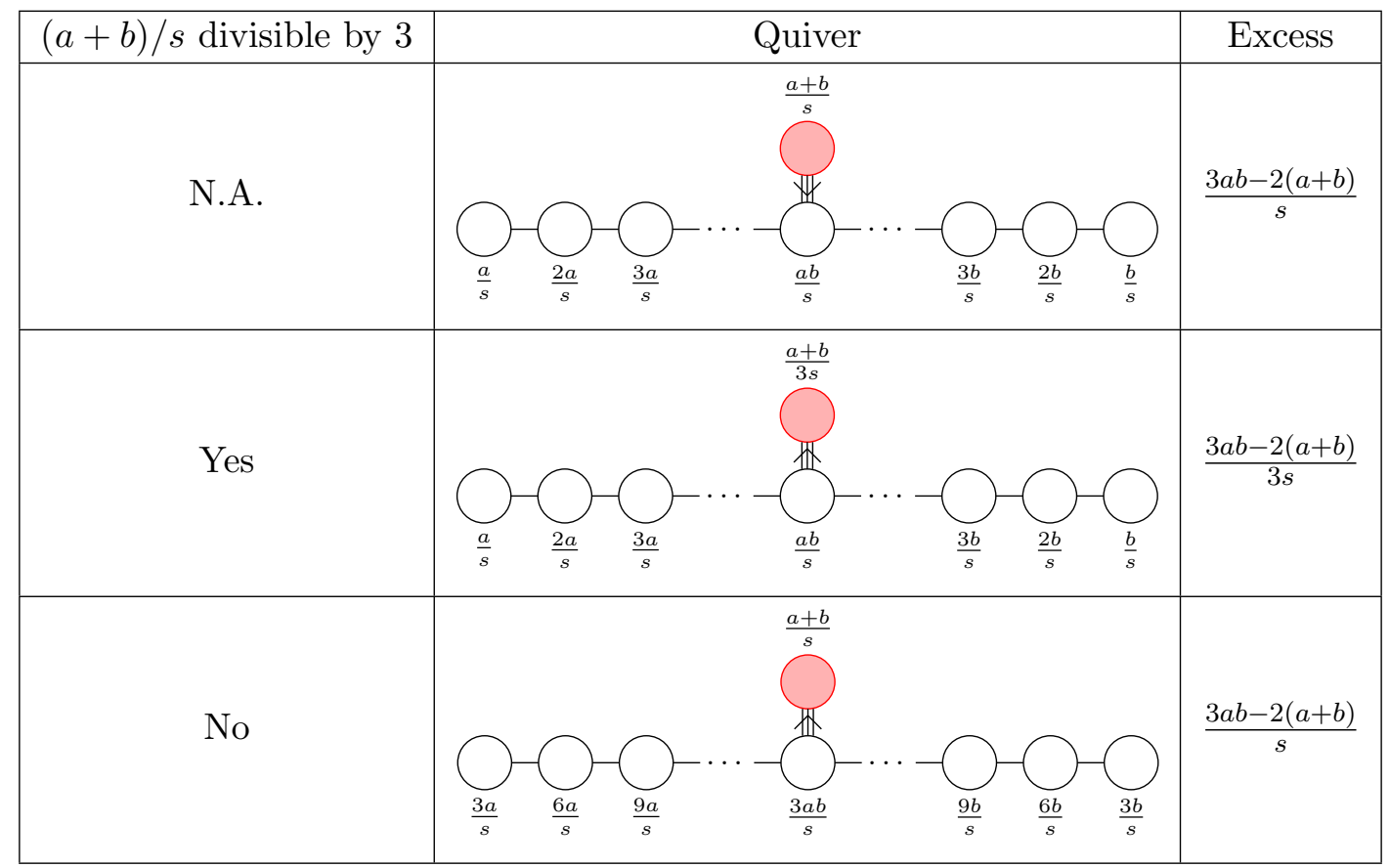

Table 13. Exotic minimally unbalanced quivers with $G=\mathrm{SU}(n), n=a+b$ and a triple laced edge. $s$ is the greatest common divisor of $a$ and $b$. 


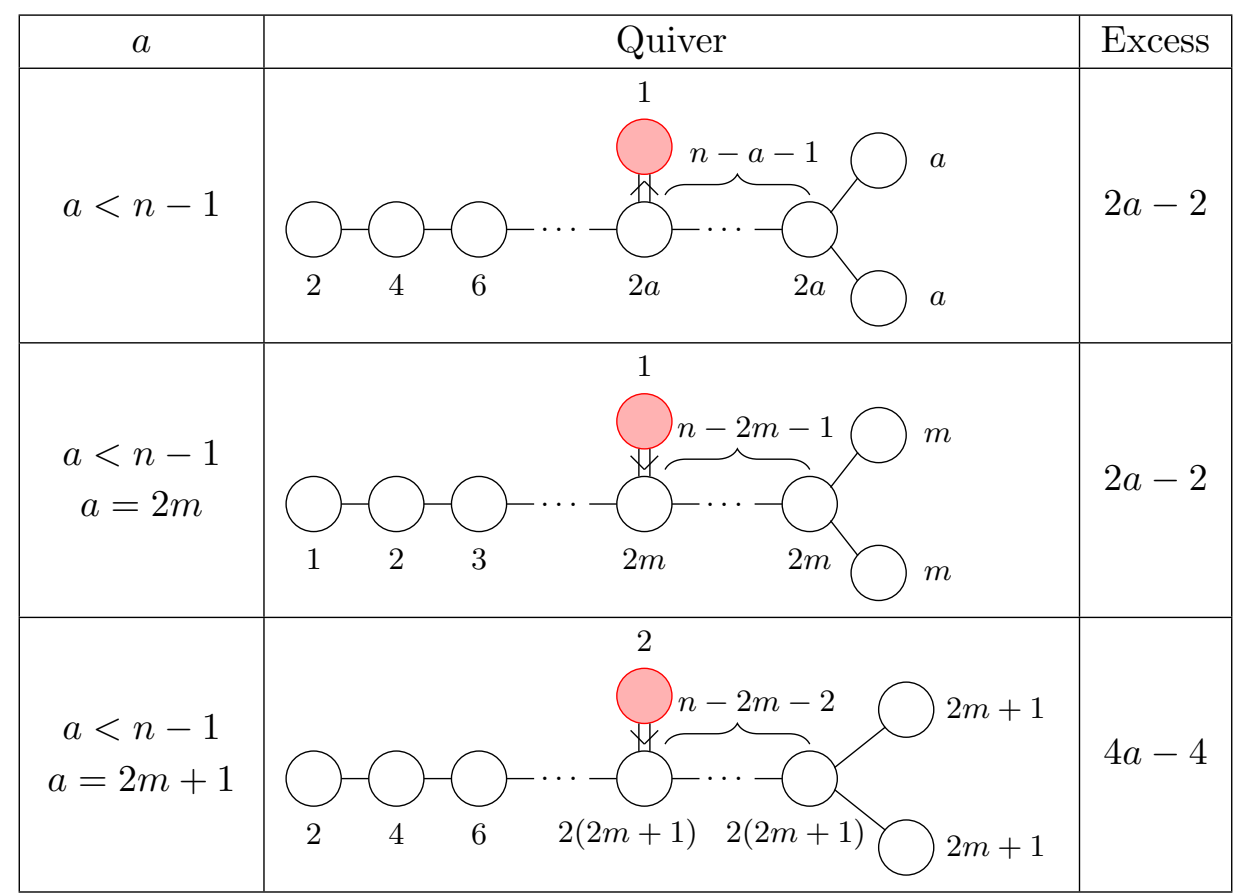

Table 14. Exotic minimally unbalanced quivers with $G=\operatorname{SO}(2 n)$ and a double laced edge.

\subsection{Exotic minimally unbalanced quivers with $G$ of type $E_{n}$}

In this subsection, we classify all exotic minimally unbalanced quivers of $E_{n}$-type with the unbalanced node connected to the balanced part of the quiver by a non-simply laced edge.

\subsubsection{Exotic minimally unbalanced quivers with $G$ of type $E_{6}$}

We start by showing the results for all exotic minimally unbalanced quivers with $G=E_{6}$ and with the unbalanced node connected by a double and triple laced edge. The former quivers are collected in table 18 and the latter in table 19, respectively. Note that, within the tables, a further distinction involves the orientation of the non-simply laced edge and the position of the unbalanced node. The orientation is always considered with respect to the unbalanced node.

\subsubsection{Exotic minimally unbalanced quivers with $G$ of type $E_{7}$}

We repeat the same program for exotic minimally unbalanced quivers with $G=E_{7}$. First, we distinguish whether the unbalanced node is connected via a double laced edge directed; outwards from the unbalanced node, table 20, or inwards with respect to the unbalanced node, table 21. Next, we turn to the quivers with the unbalanced node connected via a triple laced edge. The resulting quivers with a triple laced edge pointing outwards and inwards with respect to the unbalanced node are reported in table 22 and 23, respectively.

\subsubsection{Exotic minimally unbalanced quivers with $G$ of type $E_{8}$}

Finally, we present all exotic minimally unbalanced quivers with $G=E_{8}$. Tables 24 and 25 contain quivers with the unbalanced node connected by a double laced edge pointing 


\begin{tabular}{|l|l|l|}
\hline & $a$ \\
$a=n$ &
\end{tabular}

Table 15. Exotic minimally unbalanced quivers with $G=\operatorname{SO}(2 n)$ and a double laced edge.

outwards and inwards from the unbalanced node, respectively. Tables 26 and 27 collect quivers with a triple laced edge directed outwards and inwards from the unbalanced node, respectively.

\section{Non-simply laced minimally unbalanced quivers with unbalanced node connected by a non-simply laced edge}

Let us emphasize the main criteria for the classification of minimally unbalanced quivers:

- Balanced subset of nodes must form a single Dynkin diagram of finite type

- The unbalanced node must connect to a single node of the Dynkin diagram with either a simple, double or a triple laced edge ${ }^{20}$

It follows from this criterion that the remaining part of the classification concerns quivers with two non-simply laced edges. Analogically to section 6 we term the following part of

\footnotetext{
${ }^{20}$ By relaxing the second criterion one can produce very exotic minimally unbalanced quivers like the A-type Ring quivers in appendix B. The motivation for presenting the quivers in appendix B is that the unbalanced node connects to the adjoint nodes which results in a simpler moduli space compared to a generic cases where the unbalanced node connects to any two arbitrary nodes of a Dynkin diagram.
} 


\begin{tabular}{|c|c|c|c|c|c|}
$a<n-1$ \\
$a=2 m$ \\
$a=2 m+1$
\end{tabular}

Table 16. Exotic minimally unbalanced quivers with $G=\mathrm{SO}(2 n)$ and a triple laced edge.

the classification exotic classification of minimally unbalanced quivers of non-simply laced theories. In the following the quivers are divided into categories based on:

- Dynkin diagram type of the balanced part of the quiver

- Type of the non-simply laced edge:

- Double Edge

- Triple Edge

- Orientation of the non-simply laced edge that connects the unbalanced node. The orientation is considered with respect to the unbalanced node:

- Outwards

- Inwards

- Position of the unbalanced node (with respect to the balanced sub-quiver)

We emphasize that all quivers in this section are shown in their basic form such that the ranks are the lowest possible integers. All other quivers that are also minimally unbal- 


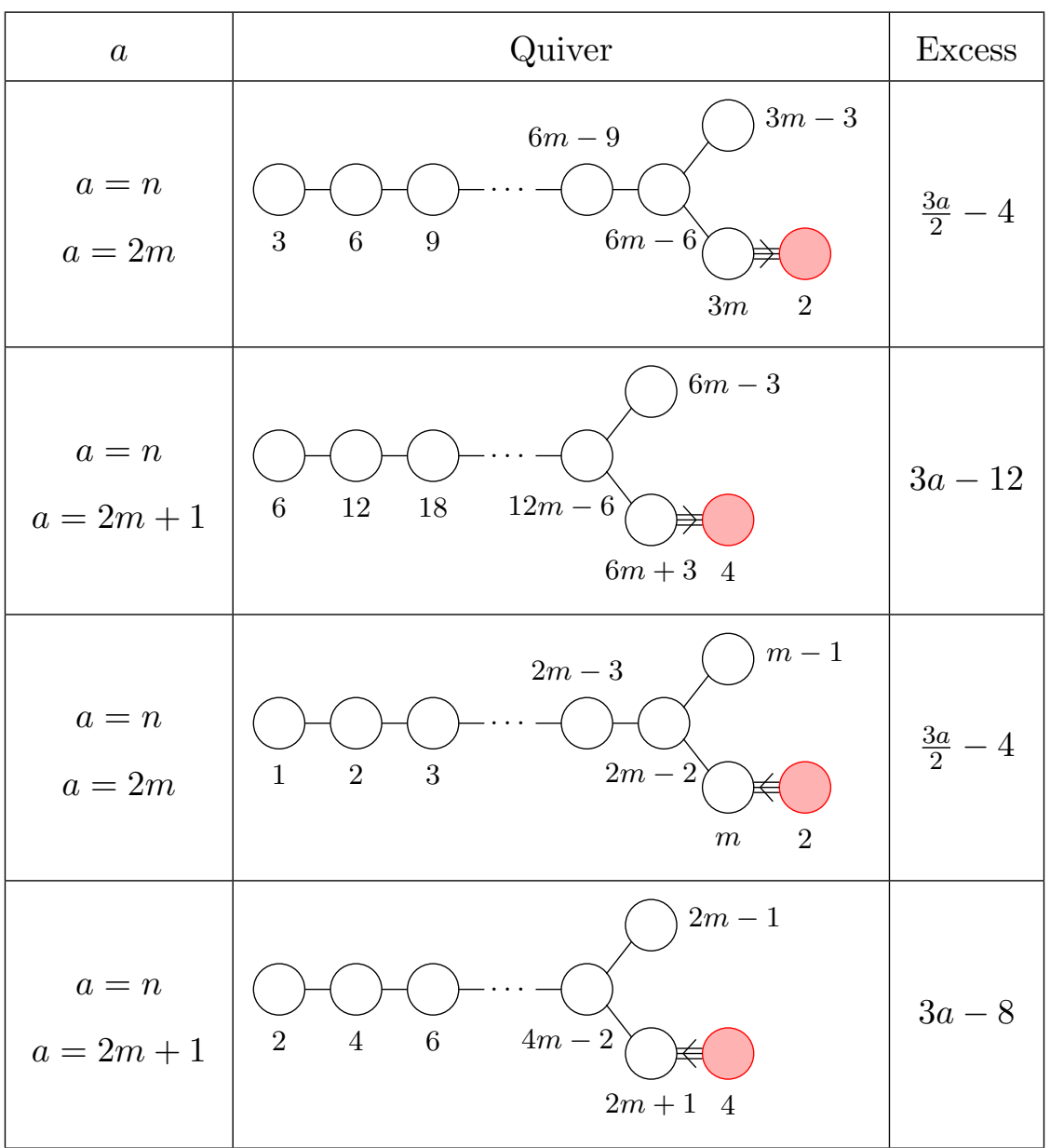

Table 17. Exotic minimally unbalanced quivers with $G=\mathrm{SO}(2 n)$ and a triple laced edge.

anced and have the same global symmetry $G$ on their Coulomb branch can be obtained by multiplying the basic canonical forms by an integer number.

\subsection{Exotic minimally unbalanced quivers with $G$ of type $B_{n}$}

Let us start with exotic minimally unbalanced quivers with $G=\mathrm{SO}(2 n+1)$ and with the unbalanced node connected to the rest of the quiver via a double laced edge. The eight resulting cases are summarized in table 28. Analogically, table 29 contains all quivers which contain an extra triple laced edge between the unbalanced node and the rest of the quiver.

\subsection{Exotic minimally unbalanced quivers with $G$ of type $C_{n}$}

Next, we present the four cases of exotic minimally unbalanced quivers with $G=C_{n}$ and the unbalanced node connected via a double laced edge. The results are summarized in table 30 . In table 31 we collect the remaining four quivers where the unbalanced node is connected via a triple laced edge. 


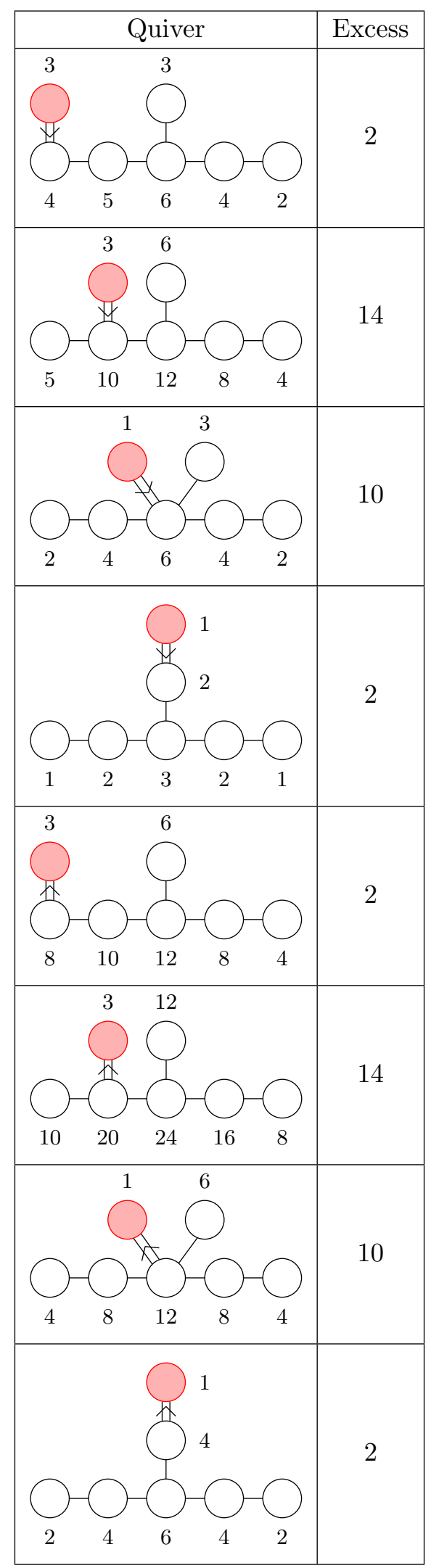

Table 18. Exotic minimally unbalanced quivers of $E_{6}$-type with a double laced edge. 


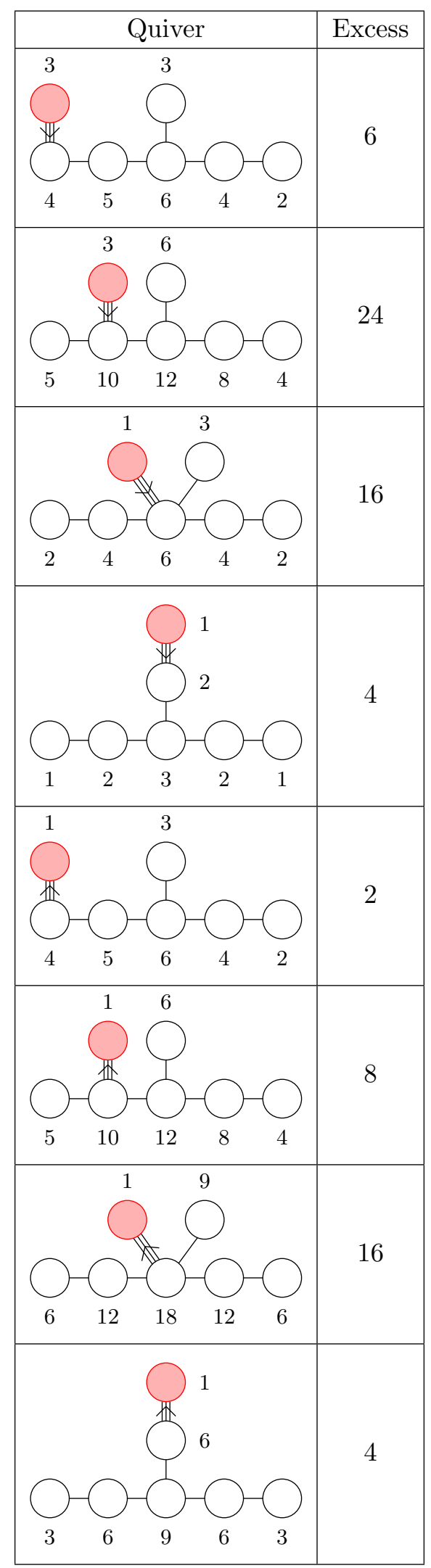

Table 19. Exotic minimally unbalanced quivers of $E_{6}$-type with a triple laced edge. 


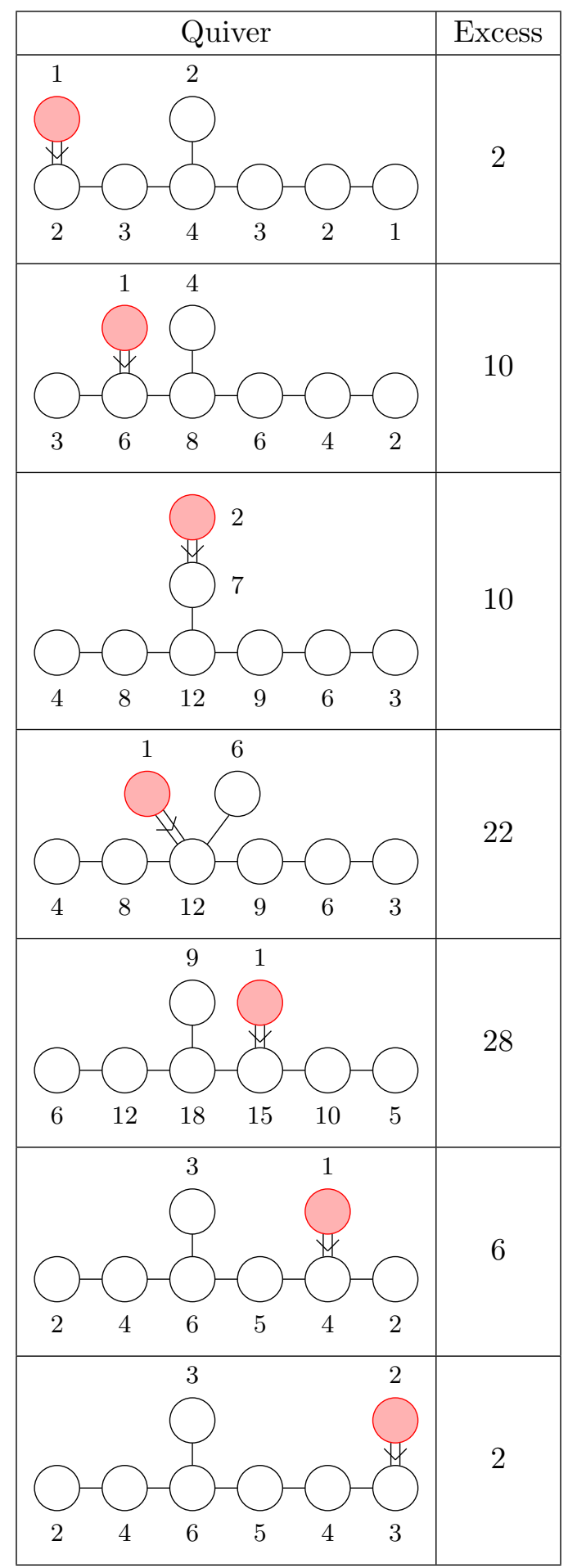

Table 20. Exotic minimally unbalanced quivers of $E_{7}$-type with outward double laced edge. 


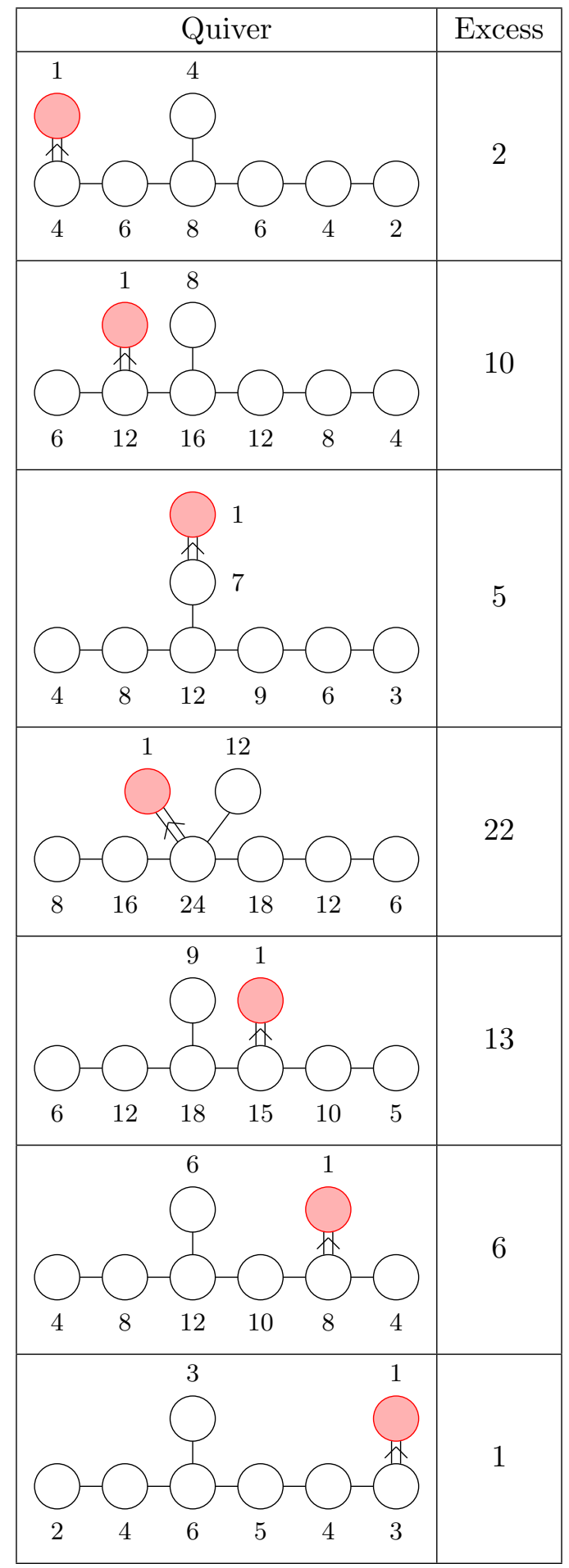

Table 21. Exotic minimally unbalanced quivers of $E_{7}$-type with inward double laced edge. 


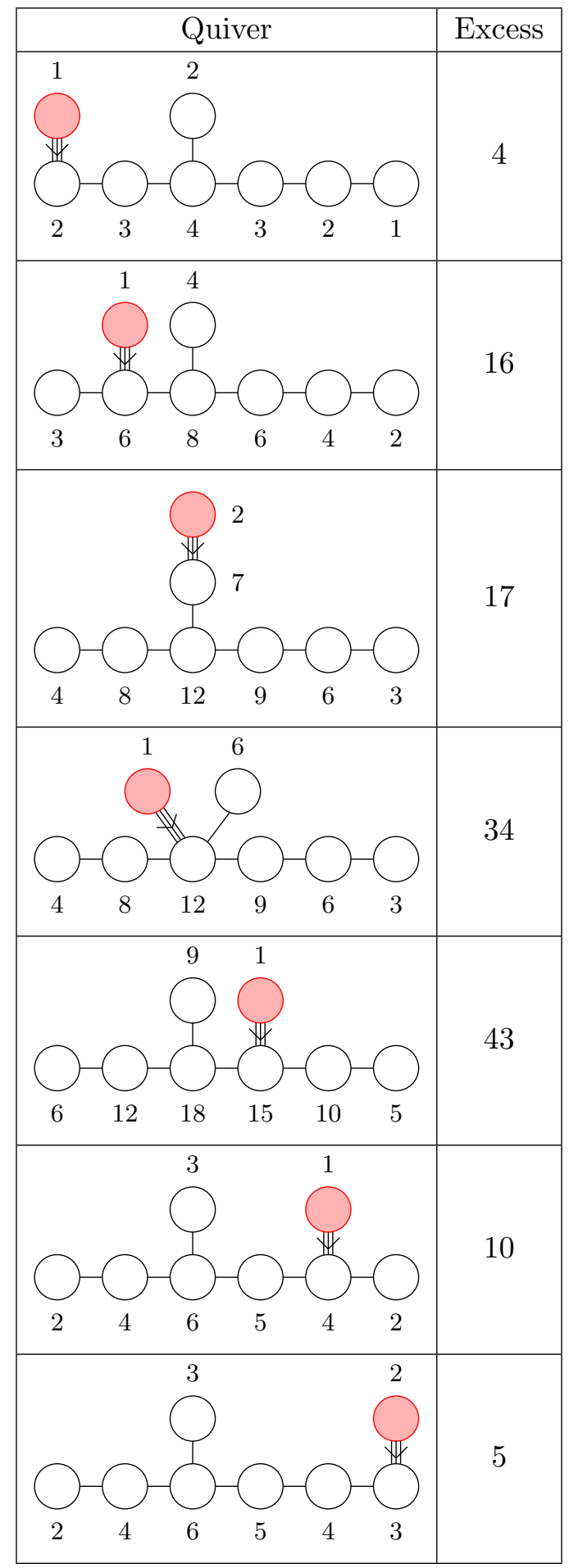

Table 22. Exotic minimally unbalanced quivers of $E_{7}$-type with outward triple laced edge. 


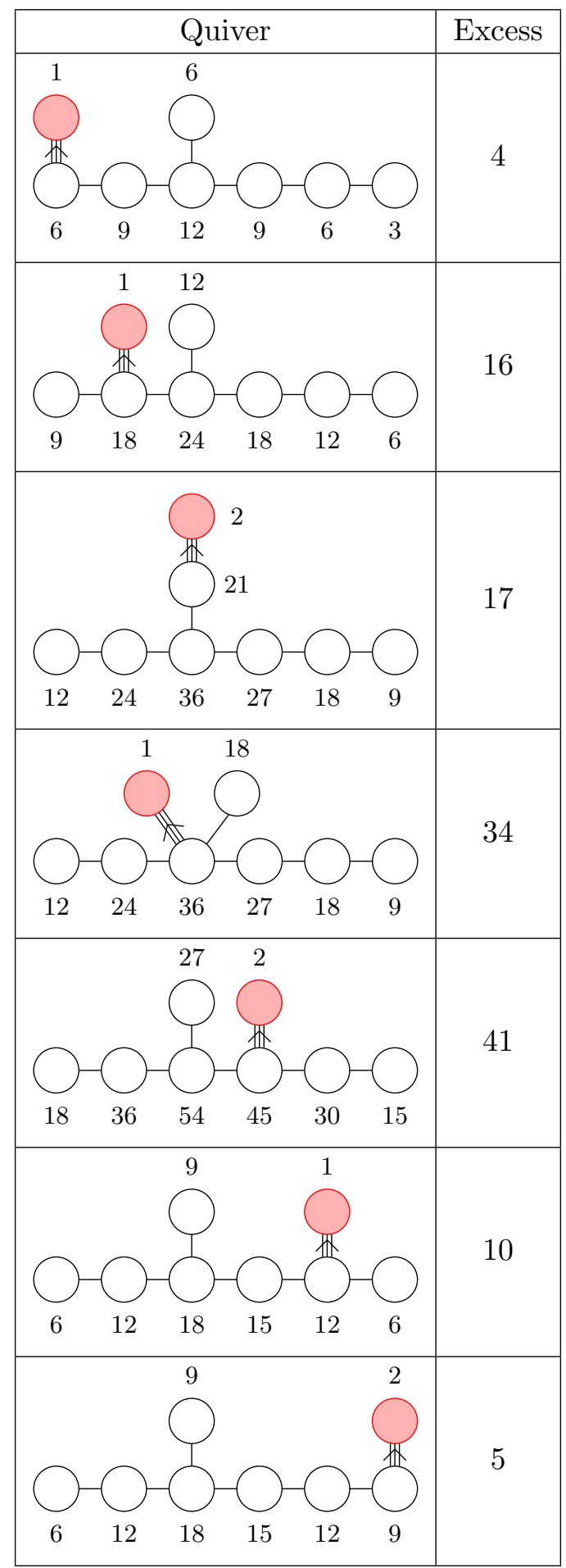

Table 23. Exotic minimally unbalanced quivers of $E_{7}$-type with inward triple laced edge. 


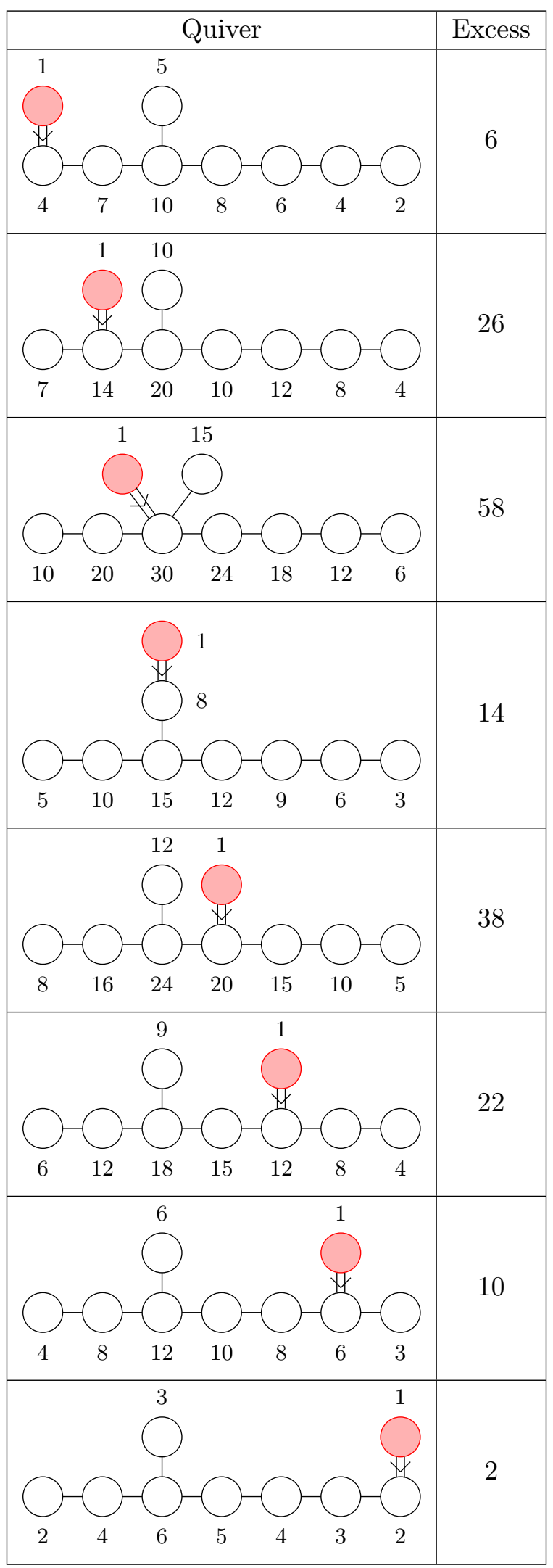

Table 24. Exotic minimally unbalanced quivers of $E_{8}$-type with outward double laced edge. 


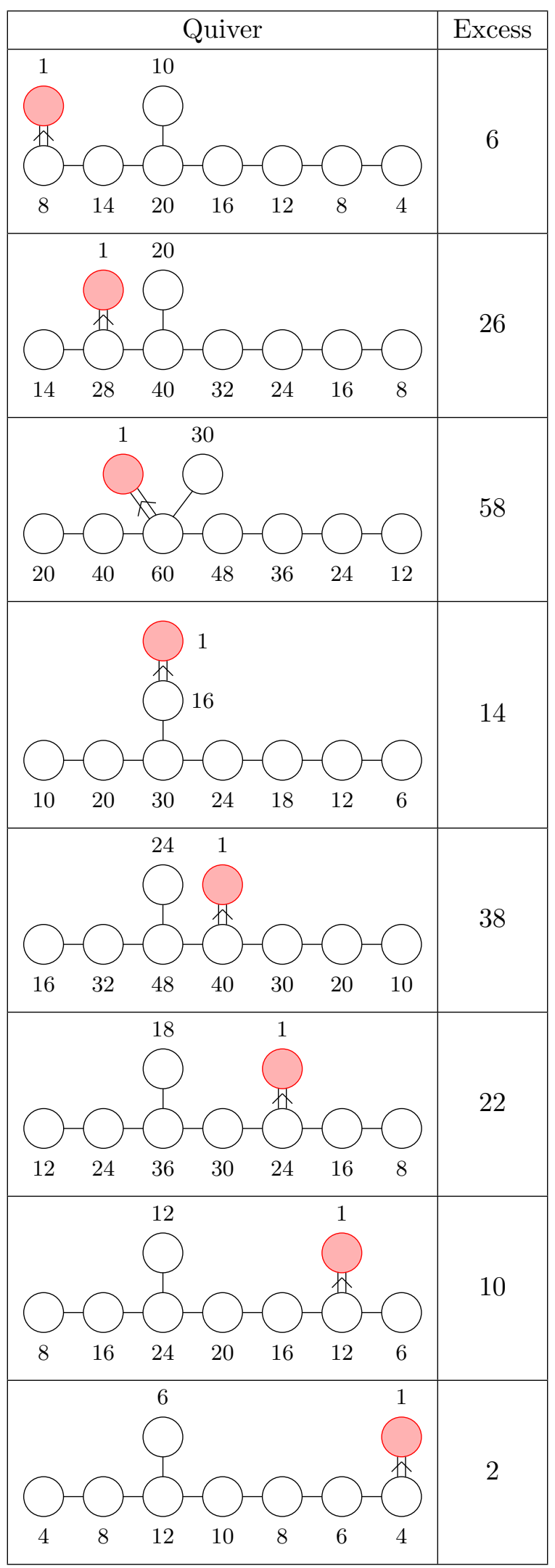

Table 25. Exotic minimally unbalanced quivers of $E_{8}$-type with inward double laced edge. 


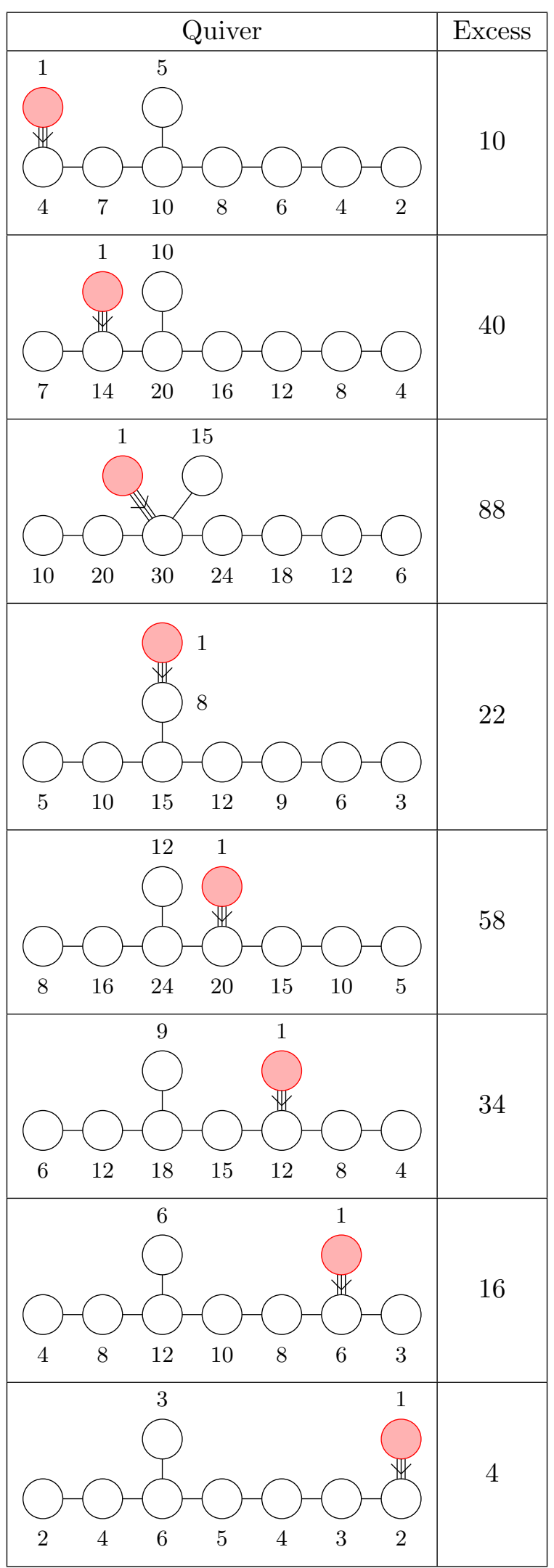

Table 26. Exotic minimally unbalanced quivers of $E_{8}$-type with outward triple laced edge. 


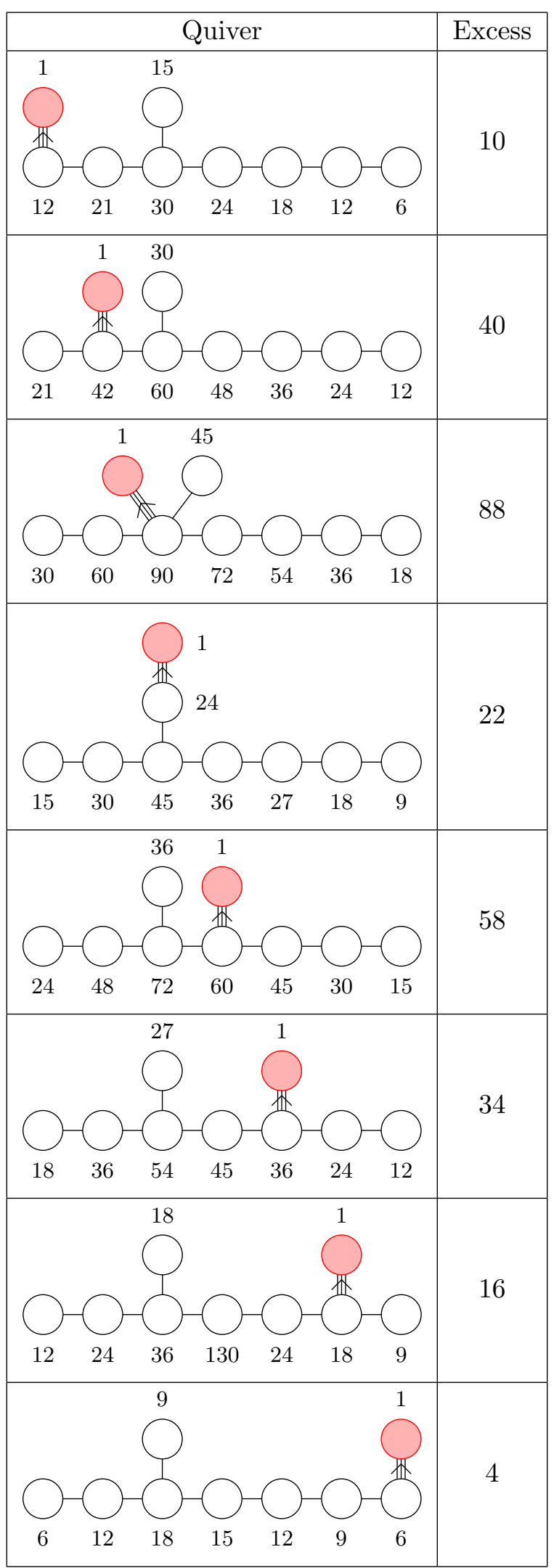

Table 27. Exotic minimally unbalanced quivers of $E_{8}$-type with inward triple laced edge. 


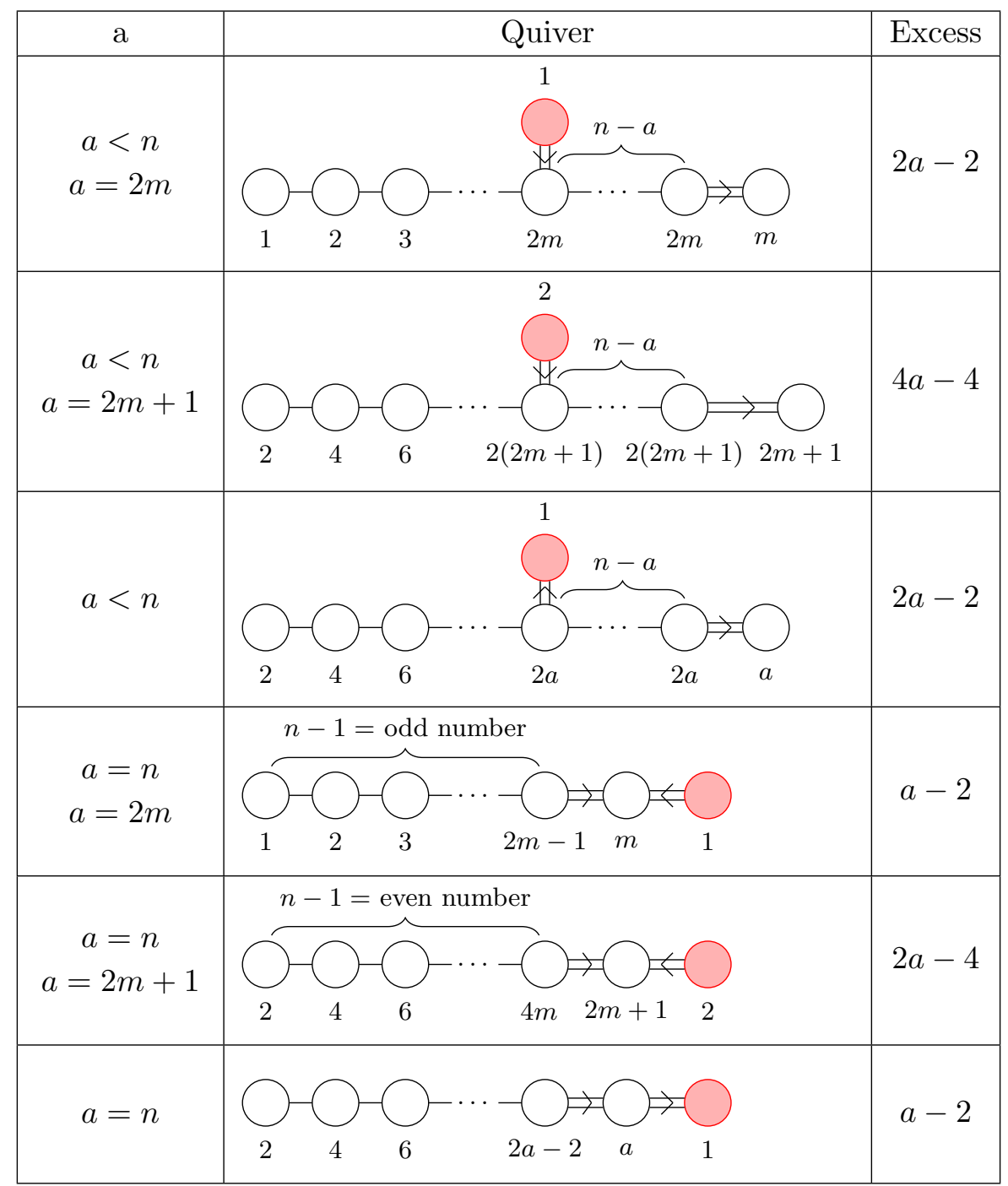

Table 28. Exotic minimally unbalanced quivers with $G=\mathrm{SO}(2 n+1)$ and an extra double laced edge.

\subsection{Exotic minimally unbalanced quivers with $G$ of type $F_{4}$}

In this subsection we present all exotic minimally unbalanced quivers with $G=F_{4}$. Quivers with an extra double laced edge are summarized in table 32. All quivers with extra triple laced edge are collected in table 33 .

\subsection{Exotic minimally unbalanced quivers with $G$ of type $G_{2}$}

Finally, we collect all exotic minimally unbalanced quivers with $G=G_{2}$ and with the unbalanced node attached via a double or a triple laced edge in tables 34 and 35, respectively.

This completes the classification of minimally unbalanced quivers for which the balanced subset of nodes forms a single finite Dynkin diagram. Quivers found in sections 4 and 5 have Coulomb branches minimally generated by two kinds of operators: 


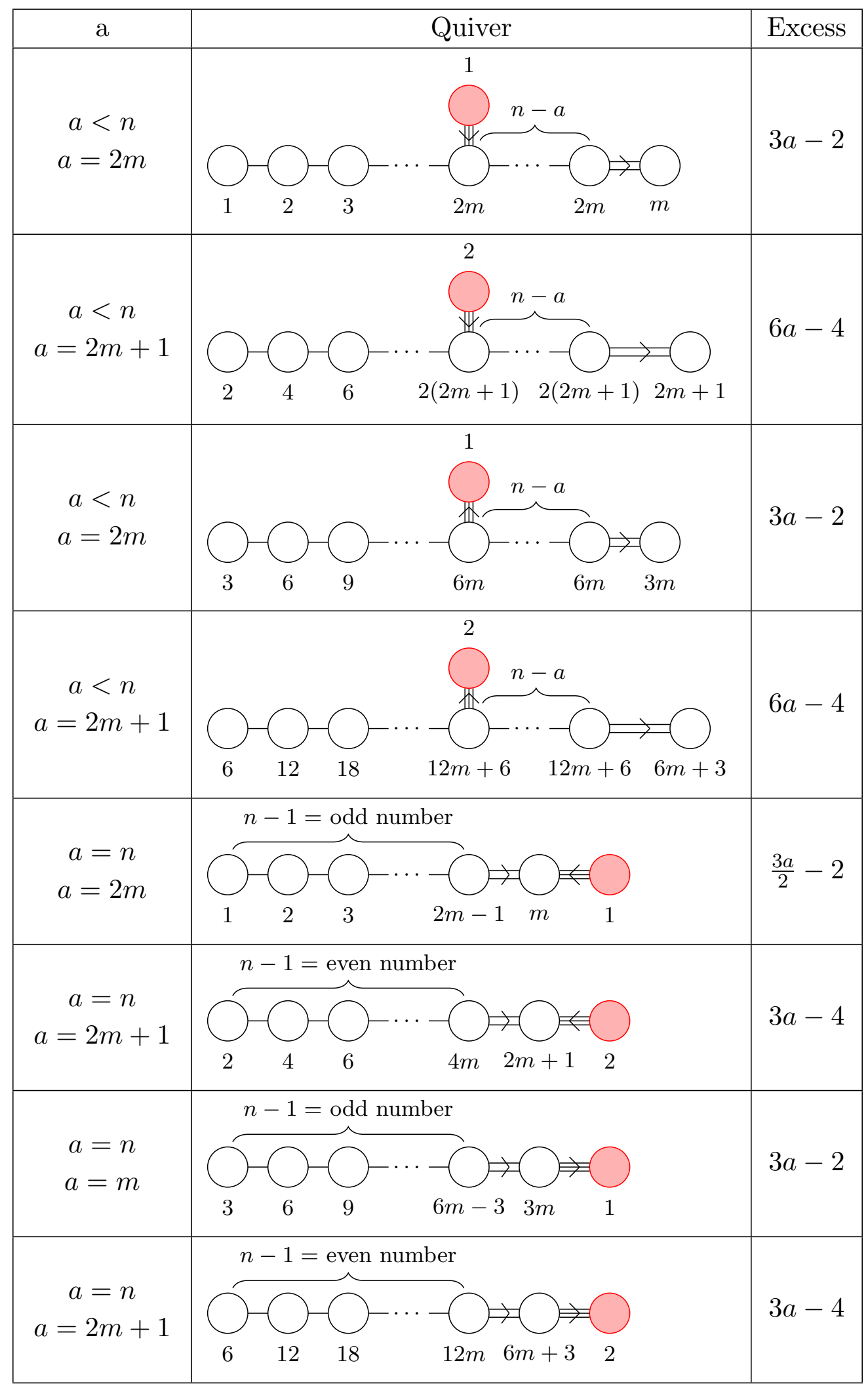

Table 29. Exotic minimally unbalanced quivers with $G=\mathrm{SO}(2 n+1)$ and an extra triple laced edge. 


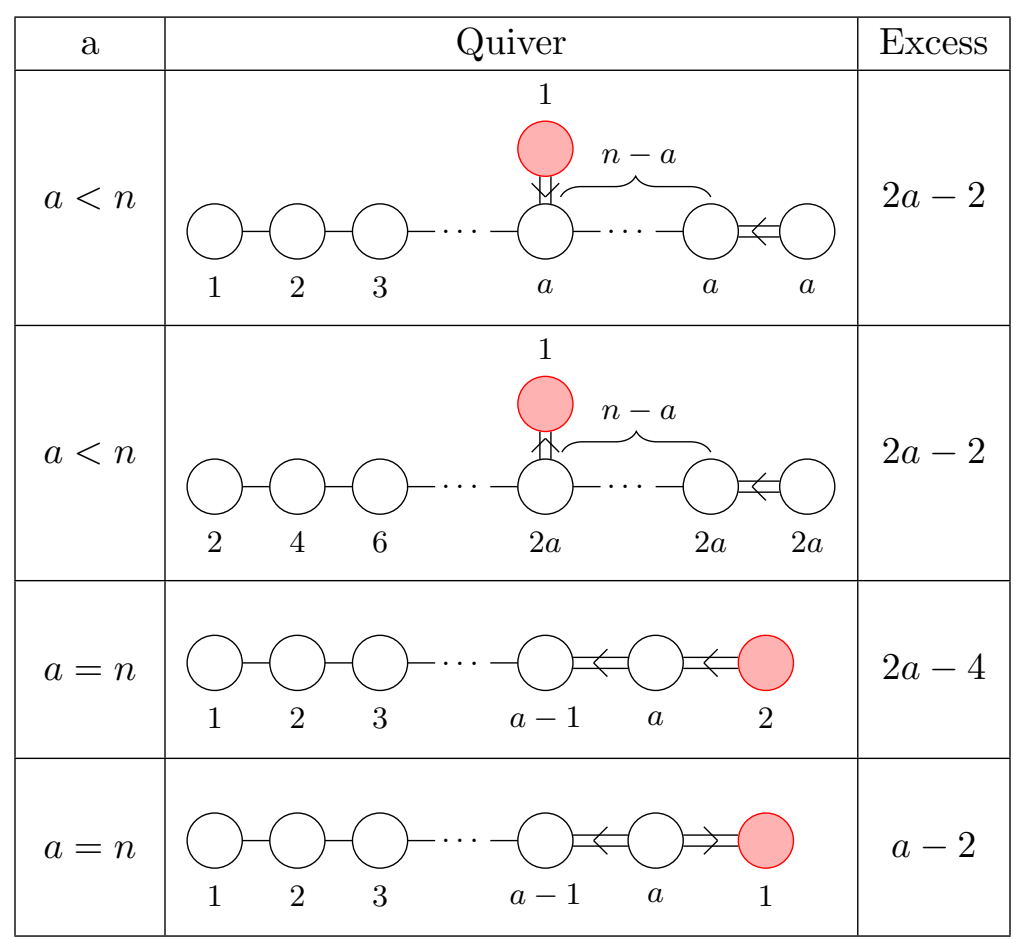

Table 30. Exotic minimally unbalanced quivers with $G=\operatorname{Sp}(2 n)$ and an extra double laced edge.

- Operators in the adjoint of the isometry group $G$ (corresponding to the balanced Dynkin diagram). These operators appear at order $t^{2}$ in the Hilbert series.

- Extra operators in the representation that corresponds to the Dynkin node where the unbalanced extra node attaches (and its complex conjugate representation). In the Hilbert series, these appear at order of $t^{k}$, where $k$ is determined by the excess (imbalance of the unbalanced node). In particular, $k=2+e$, where $e$ is the excess.

Section 6 and 7 contain exotic minimally unbalanced quivers. The Coulomb branches of these quivers are, strictly speaking, not minimally generated but have additional generators at higher orders. Although these spaces correspond to moduli spaces of gauge theories with eight supercharges, this is the first time most of such quivers appear. The full gauge theoretic examination of such theories is yet to be done.

\section{Conclusions and prospects}

In this paper we develop and present a full classification of all minimally unbalanced quiver gauge theories with the global isometry on the Coulomb branch that corresponds to a single finite Dynkin diagram. This concurrently provides a classification of hyperkähler cones which appear as moduli spaces of supersymmetric gauge theories with 8 supercharges in various dimensions. Minimally unbalanced quivers with $A$-type global symmetry form a two parameter family described by $a$ and $b$. Minimally unbalanced theories with global symmetry of $B C D$-type are classified based on the position of the unbalanced node and 


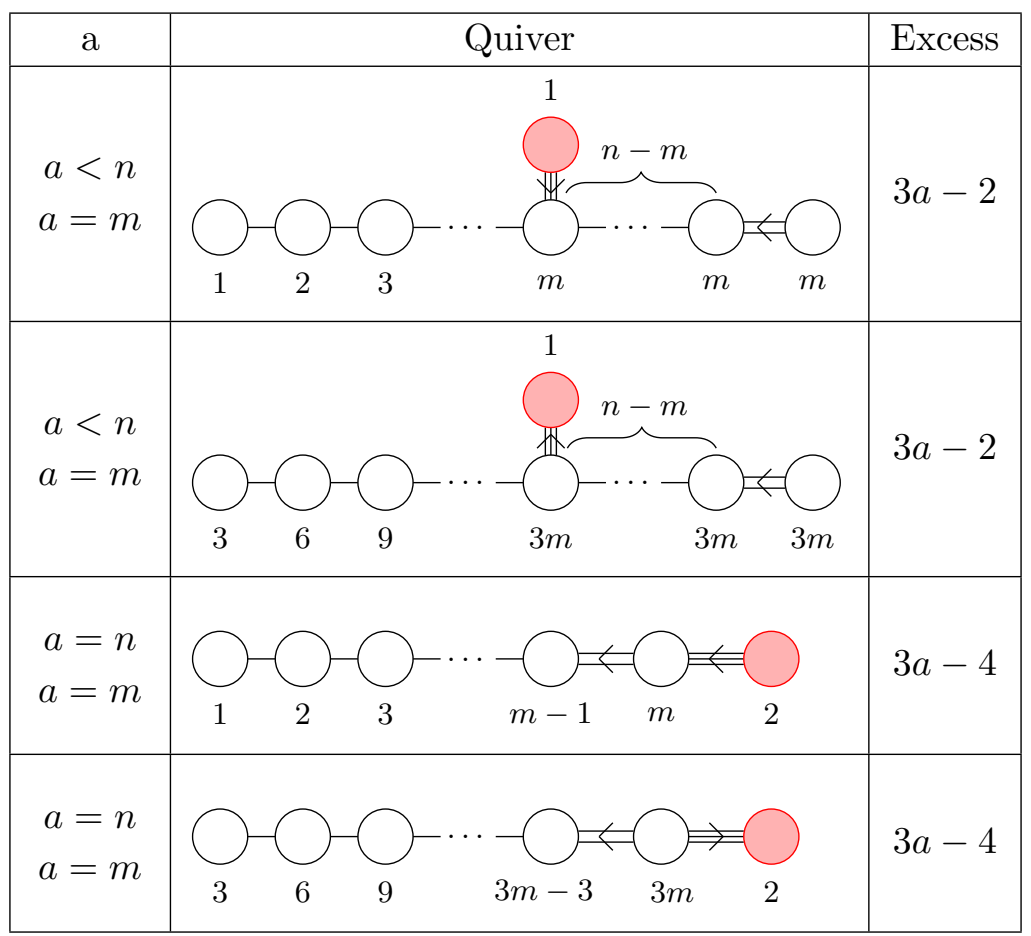

Table 31. Exotic minimally unbalanced quivers with $G=\operatorname{Sp}(2 n)$ and an extra triple laced edge.

the total number of balanced nodes. Minimally unbalanced quivers with exceptional global symmetry (i.e. of $E F G$-type) are found for each different case (i.e. for each possible node to which the unbalanced node is attached). In case of $E_{6}$, the number of cases reduces to 4 due to the $\mathbb{Z}_{2}$ outer automorphism invariance of the $E_{6}$ Dynkin diagram.

A complementary result to the work in this paper is the classification of minimally unbalanced quivers with global symmetry of the form:

$$
G_{\text {global }}=G_{1} \times G_{2},
$$

where $G_{1}$ and $G_{2}$ are any two Lie groups. Such extended classification is obtained by combining all pairs of minimally unbalanced quivers found in this paper. ${ }^{21}$ The extended classification is available online at https://www.dropbox.com/s/uxi30bgjis1x4u2/AUX_MU.pdf?dl=0 or for download at https://dl.dropboxusercontent.com/s/uxi30bgjis1x4u2/AUX_MU.pdf?dl=0. ${ }^{22}$

A possible direction for future research is the classification of unbalanced quivers with $N$ unbalanced nodes, where $N>1$. In such scenario, the global symmetry takes the form:

$$
G_{\text {global }}=\prod_{i} G_{i} \times \mathrm{U}(1)^{N-1},
$$

where $G_{i}$ are the groups corresponding to the Dynkin sub-diagrams formed by the subset of balanced nodes. The number of the U(1) Abelian factors in the global symmetry is one less than the number of unbalanced nodes. Quivers with more than one unbalanced node appear in various contexts in the study of $5 d$ and $6 d$ Higgs branches [18, 21, 45].

\footnotetext{
${ }^{21}$ Combination means an attaching of two minimally unbalanced quivers via a common unbalanced node.

${ }^{22}$ Alternatively, the extended classification is available as per request by email.
} 


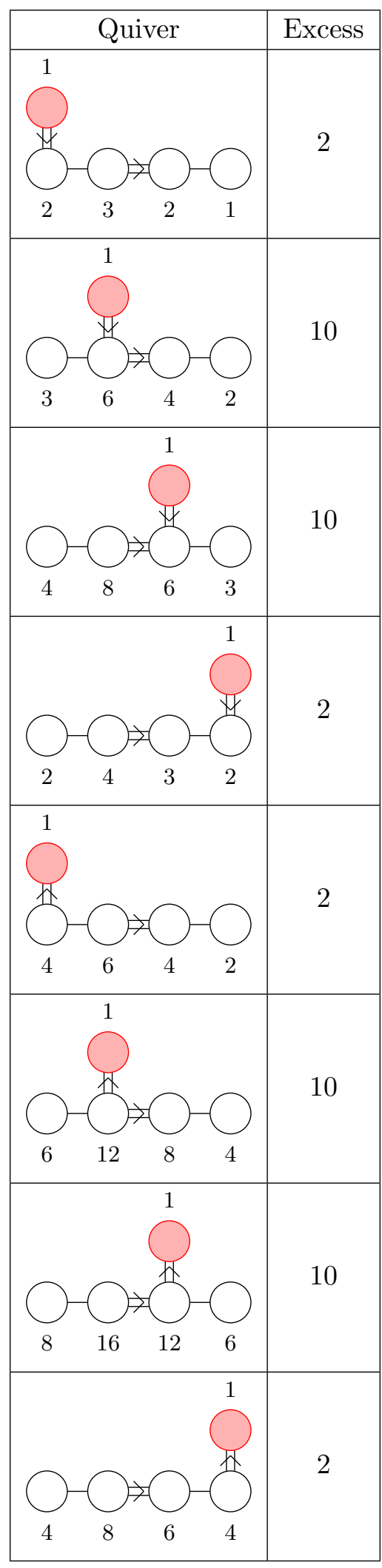

Table 32. Exotic minimally unbalanced quivers with $G=F_{4}$ and an extra double laced edge. 


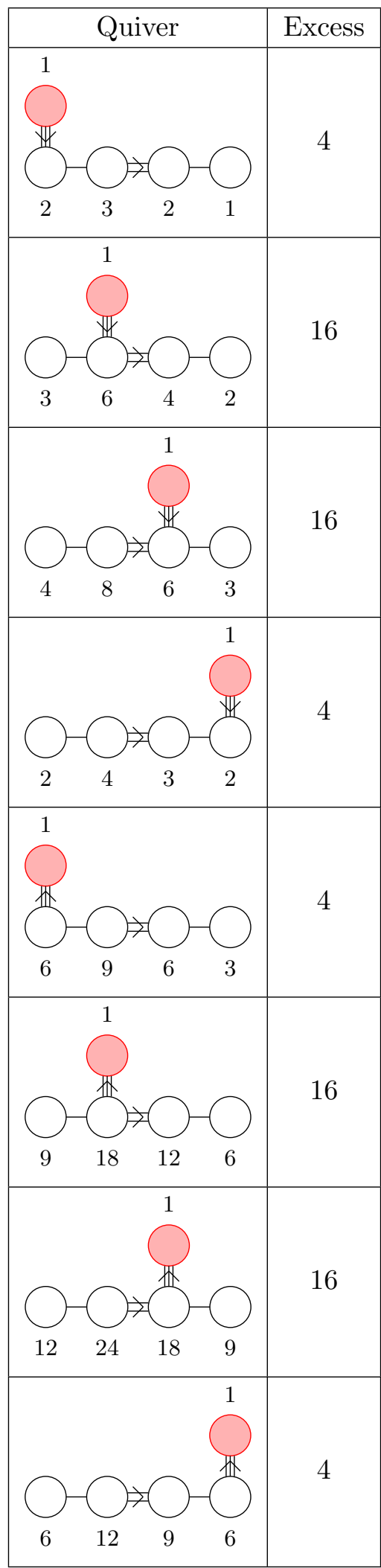

Table 33. Exotic minimally unbalanced quivers with $G=F_{4}$ and an extra triple laced edge. 


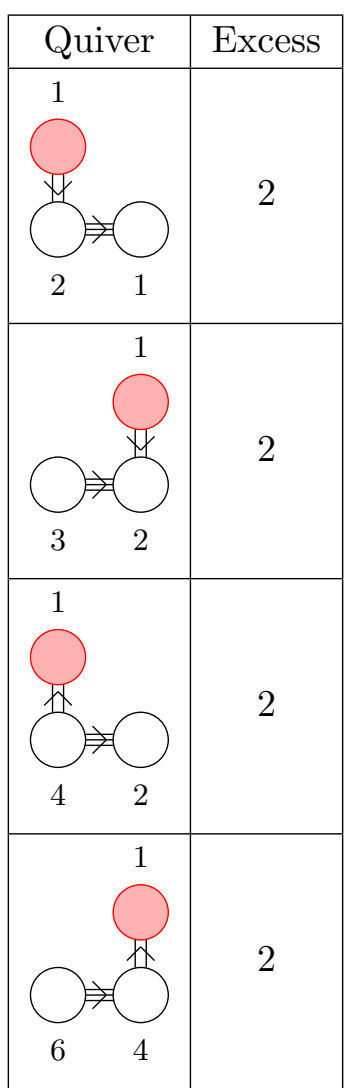

Table 34. Exotic minimally unbalanced quivers with $G=G_{2}$ and an extra double laced edge.

In the classification of this paper, we find a raft of quiver theories that are not studied in any existing literature. This opens a large and possibly fructiferous domain for extensive future investigations. Quaerite et invenietis ordinem.

\section{Acknowledgments}

S.C. is supported by an EPSRC DTP studentship EP/M507878/1. A.H. is supported by STFC Consolidated Grant ST/J0003533/1, and EPSRC Programme Grant EP/ K034456/1. A.Z. would like to extend his gratitude to Rudolph Kalveks for enlightening discussions. A.Z. would also like to extend his gratitude to Matus Plch for coming to his aid when implementing NN tools which brought to light unexpected new minimally unbalanced quivers.

\section{A The coulomb branch: monopole operators and global symmetries}

Moduli spaces of $3 d \mathcal{N}=4$ quiver gauge theories have two distinct phases known as the Coulomb branch (where the gauge group is typically broken to its maximal torus) and Higgs branch (where the gauge group of the theory is typically fully broken). The Coulomb branch (and also the Higgs branch) is a hyperKähler variety which can be described by its ring of holomorphic functions. The information about the branch is then encoded in a 


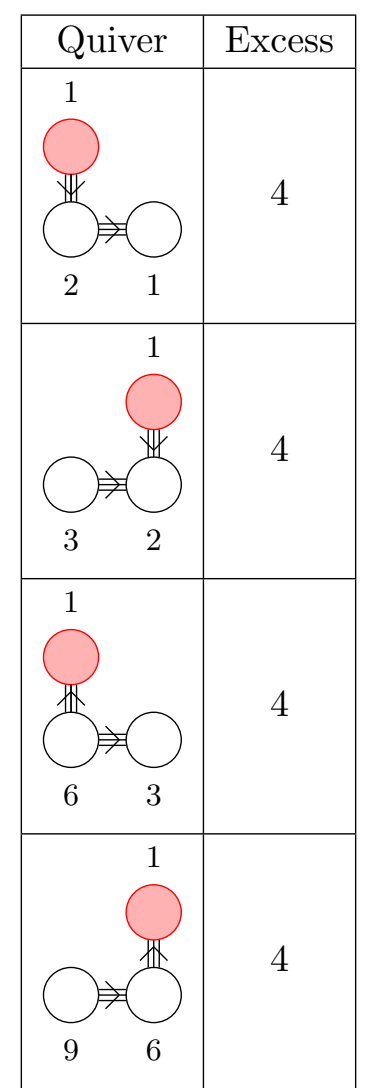

Table 35. Exotic minimally unbalanced quivers with $G=G_{2}$ and an extra triple laced edge.

Hilbert Series which succinctly enumerates holomorphic functions in the ring. A one-to-one correspondence has been observed between holomorphic functions in the moduli space and gauge invariant BPS operators in the chiral ring of the quantum field theory. In [35] an efficient method for counting these operators is proposed, namely the monopole formula:

$$
H_{G}(t, z)=\sum_{m \in \Gamma_{\hat{G}} / \mathcal{W}_{\hat{G}}} z^{J(m)} t^{\Delta(m)} P_{G}(t, m)
$$

where $G$ is the gauge group of the theory and $m$ is the magnetic charge (see [46]) which takes its value in the lattice:

$$
\Gamma_{\hat{G}}:=\left(\Gamma_{G}\right)^{*}
$$

$\left(\Gamma_{G}\right)^{*}$ is the lattice dual to the weight lattice of $G$. It defines a new weight lattice of a new group $\hat{G}$, which is considered the GNO dual of $G$ [46]. $\mathcal{W}_{\hat{G}}$ is the Weyl group of $\hat{G}$. $J(m)$ denotes the topological charge counted by the $z$ fugacity. The dressing factor $P_{G}$ is a generating function for Casimir invariants of the unbroken gauge group.

$\Delta(m)$ is the conformal dimension which coincides with the R-charge of the monopole operators. We quote the result for the conformal dimension, as it was obtained using radial 
quantization in [32]:

$$
\Delta(m)=-\sum_{\alpha \in \Delta_{+}}|\alpha(m)|+\frac{1}{2} \sum_{i=1}^{n} \sum_{\rho_{i} \in R_{i}}\left|\rho_{i}(m)\right| .
$$

The two terms of the conformal dimension formula account for vector multiplets and hypermultiplet contributions, respectively. $\Delta_{+}$is the set of positive roots of the gauge group. Hypermultiplets transform in representations $R_{i}$ with weights $\rho_{i}$.

An approach that utilizes division of weight lattice into fans was introduced in [47]. For more detailed exposition of the monopole formula, see [35]. In order to treat non-simply laced quivers, a modification of the hypermultiplet contribution of the conformal dimension introduced in [39] takes the following form:

$$
\frac{1}{2}\left|\rho_{i}(m)\right| \rightarrow \frac{1}{2} \sum_{j=1}^{N_{1}} \sum_{k=1}^{N_{2}}\left|\lambda m_{j}^{(1)}-m_{k}^{(2)}\right|
$$

where $\rho_{i}$ is the irrep corresponding to the hypermultiplets assigned to the edge between two nodes $\mathrm{U}\left(N_{1}\right)$ and $\mathrm{U}\left(N_{2}\right) . \quad \lambda=1$ recovers the formula for the quiver when the edge is simple, $\lambda=2$ is used for a double laced edge, and finally, $\lambda=3$ for a triple laced edge. The direction of the edge points from $N_{1}$ to $N_{2} \cdot m^{(1)}$ and $m^{(2)}$ denote the magnetic fluxes for $\mathrm{U}\left(N_{1}\right)$ and $\mathrm{U}\left(N_{2}\right)$, respectively. For completeness, we show the function which enumerates the Casimir invariants of residual gauge group of $\mathrm{U}(N)$ that is left unbroken by the configuration of magnetic charges:

$$
P_{\mathrm{U}(N)}(t ; m)=\prod_{k=1}^{N} \frac{1}{\left(1-t^{2 k}\right)^{\lambda(k)(m)}} .
$$

$\lambda(k)(m)$ encodes the various configurations of the gauge symmetry braking in form of a partition. As an example, for a $\mathrm{U}(2)$ gauge symmetry and magnetic charges $m=\left(m_{1}, m_{2}\right)$ the dressing factor is:

$$
P_{\mathrm{U}(2)}\left(t ; m_{1}, m_{2}\right)=\left\{\begin{array}{lll}
\frac{1}{(1-t)\left(1-t^{2}\right)} & \text { if } & m_{1}=m_{2} \\
\frac{1}{(1-t)(1-t)} & \text { if } & m_{1} \neq m_{2} .
\end{array}\right.
$$

In order to use the monopole formula, there are certain restrictions for the conformal dimension which translate into the balancing of the quiver nodes. Firstly, for ADE quivers, the excess (or balance) of a particular $\mathrm{U}\left(N_{i}\right)$ gauge node is defined as [41]:

$$
\operatorname{Excess}_{\mathrm{ADE}}(i)=\sum_{j \in \text { adjecent nodes }} N_{j}-2 N_{i} .
$$

A quiver is said to be fully balanced if the excess of all its nodes is zero. If one or more nodes in the quiver have positive excess the quiver is said to be positively balanced. In case of a quiver with a single node with excess of 1 or larger, we term the quiver minimally 
unbalanced. ${ }^{23}$ The present work only concerns balanced and minimally unbalanced quivers. For balanced or minimally unbalanced theories the conformal dimension satisfies $\Delta(m)>0$ for all $m \in \Gamma_{\hat{G}}$ which guarantees that the monopole formula can be applied to calculate the Coulomb branch of the moduli space. ${ }^{24}$

The global symmetry of the Coulomb branch is determined by the operators with $\Delta=1$. From the quiver one can quickly write a set of operators with $\Delta=1$ such that they correspond to the roots of the Dynkin diagram formed by nodes that are balanced. Extra operators with $\Delta=1$ might exist, which would enhance the global symmetry. In the previous pages we are restricted to quivers where only one node is unbalanced, and the remaining nodes form the Dynkin diagram of either a classical or an exceptional Lie algebra.

The Higgs branch of $5 \mathrm{~d}$ theories at infinite coupling is given by the Coulomb branch of a 3d quiver. Physically, we can motivate this with a use of $3 \mathrm{~d}$ mirror symmetry [4] and the presence of 8 supercharges in both theories. Considering a reduction of the $5 \mathrm{~d}$ SCFT on a torus leads to a $3 \mathrm{~d}$ Higgs branch that is unchanged thanks to the amount of supersymmetry. In addition, many $3 \mathrm{~d}$ theories have mirror duals for which the Coulomb and Higgs branch are exchanged. In general a dual theory can lack a Lagrangian description, however, it was argued in [23] that specific class of $5 \mathrm{~d}$ theories described by intersecting D5, NS5 and $(1,1)$-branes reduces to $A$-type class $S$ theories compactified on a circle. It was further argued that reducing class $S$ theories on circle to 3d leads to 3d SCFTs with Lagrangian mirrors whose shape is a three-legged unitary quiver. For a $\mathrm{SU}(n)$ theory with fundamental matter the bound for the number of flavors is: $N_{f}>2 n$. 5d SCFTs with enough matter belong to this class. This is a strong motivation for the approach of this paper.

\section{B Very exotic minimally unbalanced (ring) quivers with $G$ of type $A_{n}$}

Carrying out the calculation for a part of the classification, the neural network (NN), working solely with graph theoretical knowledge, produced some peculiar quivers. Among them are quivers included as a caveat in this section. In particular, we include $A$-type ring quivers with an unbalanced node connected to the adjoint nodes of the balanced chain by two non-simply laced edges. There are three cases based on whether there are two double edges, two triple edges, or one double and one triple edge. In all cases the non-simply laced edges point outwards with respect to the unbalanced node. The results are collected in table 36 with the excess shown in a separate column. Note that in all three cases, the unbalanced node connects to the Dynkin nodes corresponding to the adjoint representation of $\mathrm{SU}(m+n+2)$.

\footnotetext{
${ }^{23}$ This differs from the notation introduced in [41] where the authors used the term for all quivers with one or more nodes of excess 1 or greater.

${ }^{24}$ In fact, there are special cases of balanced quivers with moduli spaces that are not hyperKähler varieties, hence the monopole cannot be applied. Thus, it seems that balance is necessary but not sufficient condition for a quiver to be well behaved and treatable by the currently known methods.
} 


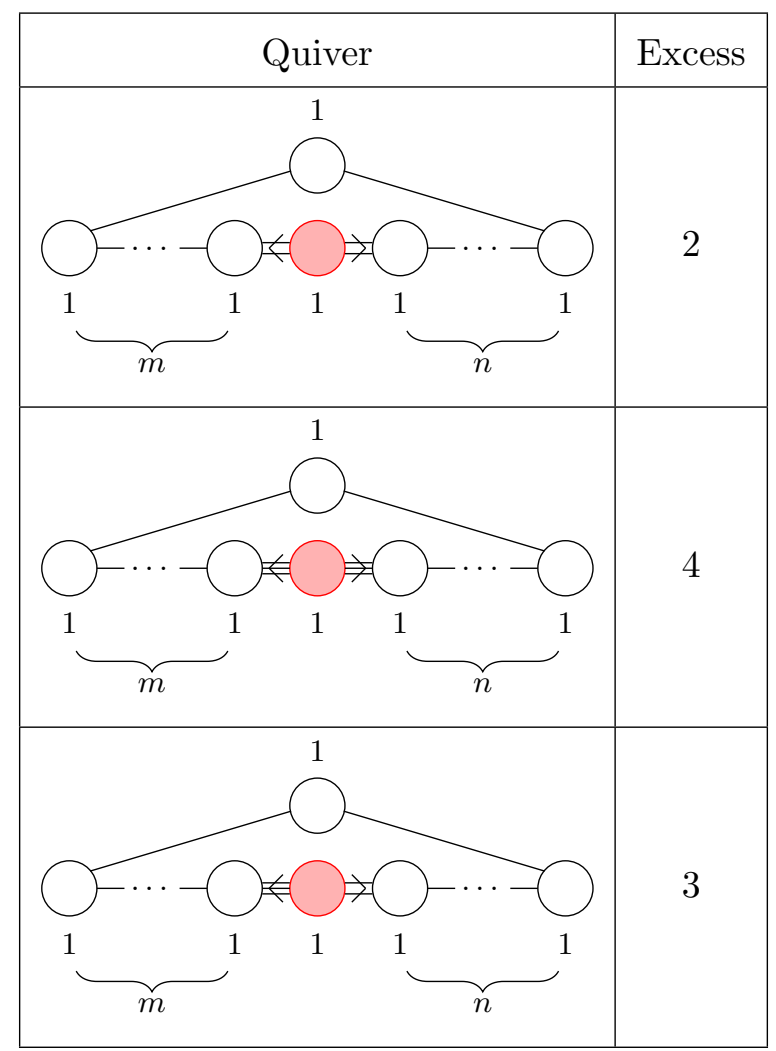

Table 36. Classification of very exotic $A$-type minimally unbalanced ring quivers with two nonsimply laced edges.

Open Access. This article is distributed under the terms of the Creative Commons Attribution License (CC-BY 4.0), which permits any use, distribution and reproduction in any medium, provided the original author(s) and source are credited.

\section{References}

[1] N. Seiberg and E. Witten, Electric-magnetic duality, monopole condensation and confinement in $N=2$ supersymmetric Yang-Mills theory, Nucl. Phys. B 426 (1994) 19 [Erratum ibid. B 430 (1994) 485] [hep-th/9407087] [INSPIRE].

[2] N. Seiberg and E. Witten, Monopoles, duality and chiral symmetry breaking in $N=2$ supersymmetric QCD, Nucl. Phys. B 431 (1994) 484 [hep-th/9408099] [INSPIRE].

[3] P.C. Argyres, M.R. Plesser and N. Seiberg, The Moduli space of vacua of $N=2$ SUSY QCD and duality in $N=1$ SUSY QCD, Nucl. Phys. B 471 (1996) 159 [hep-th/9603042] [INSPIRE].

[4] K.A. Intriligator and N. Seiberg, Mirror symmetry in three-dimensional gauge theories, Phys. Lett. B 387 (1996) 513 [hep-th/9607207] [INSPIRE].

[5] A. Hanany and E. Witten, Type IIB superstrings, BPS monopoles and three-dimensional gauge dynamics, Nucl. Phys. B 492 (1997) 152 [hep-th/9611230] [INSPIRE]. 
[6] P. Dirac, Quantised singularities in the electromagnetic field, Proc. Roy. Soc. Lond. A 133 (1931) 60 [INSPIRE] and online pdf version at http://rspa.royalsocietypublishing.org/content/133/821/60.full.pdf.

[7] A. Beauville, Symplectic singularities, Invent. Math. 139 (2000) 541 [math. AG/9903070].

[8] E. Brieskorn, Singular Elements of Semi-Simple Algebraic Groups, Actes Congres Intern. Math. 2 (1970) 279.

[9] P. Slodowy, Simple Singularities and Simple Algebraic Groups, Springer, Lect. Notes Math. 815 (1980) 1.

[10] H. Kraft and C. Procesi, On the geometry of conjugacy classes in classical groups, Comment. Math. Helv. 57 (1982) 539.

[11] P.B. Kronheimer, Instantons and the geometry of the nilpotent variety, J. Diff. Geom. 32 (1990) 473 [INSPIRE].

[12] D.H. Collingwood and W.M. McGovern, Nilpotent orbits in semisimple Lie algebras, Van Nostrand Reinhold (1993).

[13] H. Nakajima, Instantons on ALE spaces, quiver varieties, and Kac-Moody algebras, Duke Math. J. 76 (1994) 365 [inSPIRE].

[14] H. Nakajima, Towards a mathematical definition of Coulomb branches of 3-dimensional $\mathcal{N}=4$ gauge theories, I, Adv. Theor. Math. Phys. 20 (2016) 595 [arXiv:1503.03676] [INSPIRE].

[15] A. Braverman, M. Finkelberg and H. Nakajima, Towards a mathematical definition of Coulomb branches of 3 -dimensional $\mathcal{N}=4$ gauge theories, II, arXiv:1601.03586 [INSPIRE].

[16] Y. Namikawa, A characterization of nilpotent orbit closures among symplectic singularities, arXiv: 1603.06105.

[17] U. Lindström, Uses of Sigma Models, in proceedings of the 17th Hellenic School and Workshops on Elementary Particle Physics and Gravity (CORFU2017), Corfu, Greece, 2-28 September 2017, PoS(CORFU2017) 166 (2018) [arXiv:1803.08873] [INSPIRE].

[18] G. Ferlito, A. Hanany, N. Mekareeya and G. Zafrir, 3d Coulomb branch and 5d Higgs branch at infinite coupling, JHEP 07 (2018) 061 [arXiv:1712.06604] [INSPIRE].

[19] A. Hanany and N. Mekareeya, The small $E_{8}$ instanton and the Kraft Procesi transition, JHEP 07 (2018) 098 [arXiv:1801.01129] [INSPIRE].

[20] S. Cabrera and A. Hanany, Quiver Subtractions, JHEP 09 (2018) 008 [arXiv: 1803.11205] [INSPIRE].

[21] A. Hanany and G. Zafrir, Discrete Gauging in Six Dimensions, JHEP 07 (2018) 168 [arXiv: 1804.08857] [INSPIRE].

[22] D. Gaiotto and E. Witten, S-duality of Boundary Conditions In $N=4$ Super Yang-Mills Theory, Adv. Theor. Math. Phys. 13 (2009) 721 [arXiv:0807.3720] [InSPIRE].

[23] F. Benini, Y. Tachikawa and D. Xie, Mirrors of 3d Sicilian theories, JHEP 09 (2010) 063 [arXiv: 1007.0992] [INSPIRE].

[24] O. Chacaltana, J. Distler and Y. Tachikawa, Nilpotent orbits and codimension-two defects of $6 d N=(2,0)$ theories, Int. J. Mod. Phys. A 28 (2013) 1340006 [arXiv:1203.2930] [INSPIRE]. 
[25] S. Cremonesi, A. Hanany, N. Mekareeya and A. Zaffaroni, $T_{\rho}^{\sigma}(G)$ theories and their Hilbert series, JHEP 01 (2015) 150 [arXiv:1410.1548] [INSPIRE].

[26] S. Cabrera and A. Hanany, Branes and the Kraft-Procesi Transition, JHEP 11 (2016) 175 [arXiv: 1609.07798] [INSPIRE].

[27] S. Cabrera, A. Hanany and Z. Zhong, Nilpotent orbits and the Coulomb branch of $T^{\sigma}(G)$ theories: special orthogonal vs orthogonal gauge group factors, JHEP 11 (2017) 079 [arXiv: 1707.06941] [INSPIRE].

[28] S. Cabrera and A. Hanany, Branes and the Kraft-Procesi transition: classical case, JHEP 04 (2018) 127 [arXiv:1711.02378] [INSPIRE].

[29] A. Hanany and M. Sperling, Resolutions of nilpotent orbit closures via Coulomb branches of 3-dimensional $\mathcal{N}=4$ theories, JHEP 08 (2018) 189 [arXiv:1806.01890] [INSPIRE].

[30] O. Aharony, A. Hanany, K.A. Intriligator, N. Seiberg and M.J. Strassler, Aspects of $N=2$ supersymmetric gauge theories in three-dimensions, Nucl. Phys. B 499 (1997) 67 [hep-th/9703110] [INSPIRE].

[31] A. Kapustin and M.J. Strassler, On mirror symmetry in three-dimensional Abelian gauge theories, JHEP 04 (1999) 021 [hep-th/9902033] [INSPIRE].

[32] V. Borokhov, A. Kapustin and X.-k. Wu, Topological disorder operators in three-dimensional conformal field theory, JHEP 11 (2002) 049 [hep-th/0206054] [INSPIRE].

[33] V. Borokhov, A. Kapustin and X.-k. Wu, Monopole operators and mirror symmetry in three-dimensions, JHEP 12 (2002) 044 [hep-th/0207074] [INSPIRE].

[34] V. Borokhov, Monopole operators in three-dimensional $N=4 S Y M$ and mirror symmetry, JHEP 03 (2004) 008 [hep-th/0310254] [INSPIRE].

[35] S. Cremonesi, A. Hanany and A. Zaffaroni, Monopole operators and Hilbert series of Coulomb branches of $3 d \mathcal{N}=4$ gauge theories, JHEP 01 (2014) 005 [arXiv:1309.2657] [INSPIRE].

[36] A. Hanany and R. Kalveks, Highest Weight Generating Functions for Hilbert Series, JHEP 10 (2014) 152 [arXiv: 1408.4690] [INSPIRE].

[37] B. Feng, A. Hanany and Y.-H. He, Counting gauge invariants: The Plethystic program, JHEP 03 (2007) 090 [hep-th/0701063] [INSPIRE].

[38] A. Hanany and A. Pini, HWG for Coulomb branch of $3 d$ Sicilian theory mirrors, arXiv:1707.09784 [INSPIRE].

[39] S. Cremonesi, G. Ferlito, A. Hanany and N. Mekareeya, Coulomb Branch and The Moduli Space of Instantons, JHEP 12 (2014) 103 [arXiv:1408.6835] [INSPIRE].

[40] D.I. Panyushev, On spherical nilpotent orbits and beyond, Ann. Inst. Fourier 49 (1999) 1453.

[41] A. Hanany and R. Kalveks, Quiver Theories for Moduli Spaces of Classical Group Nilpotent Orbits, JHEP 06 (2016) 130 [arXiv:1601.04020] [INSPIRE].

[42] A. Hanany and R. Kalveks, Quiver Theories and Formulae for Nilpotent Orbits of Exceptional Algebras, JHEP 11 (2017) 126 [arXiv:1709.05818] [INSPIRE].

[43] J. Gray, A. Hanany, Y.-H. He, V. Jejjala and N. Mekareeya, SQCD: a geometric aperçu, JHEP 05 (2008) 099 [arXiv: 0803.4257] [inSPIRE].

[44] S. Cremonesi, G. Ferlito, A. Hanany and N. Mekareeya, Instanton Operators and the Higgs Branch at Infinite Coupling, JHEP 04 (2017) 042 [arXiv: 1505. 06302] [INSPIRE]. 
[45] A. Hanany and A. Zajac, Discrete Gauging in Coulomb branches of Three Dimensional $\mathcal{N}=4$ Supersymmetric Gauge Theories, JHEP 08 (2018) 158 [arXiv:1807.03221] [INSPIRE].

[46] P. Goddard, J. Nuyts and D.I. Olive, Gauge Theories and Magnetic Charge, Nucl. Phys. B 125 (1977) 1 [INSPIRE].

[47] A. Hanany and M. Sperling, Coulomb branches for rank 2 gauge groups in $3 d \mathcal{N}=4$ gauge theories, JHEP 08 (2016) 016 [arXiv:1605.00010] [INSPIRE]. 